\title{
In situ Investigation of Ordering Phase Transformations in FePt Magnetic Nanoparticles
}

\author{
James E. Wittig
}

Interdisciplinary Materials Science, Vanderbilt University, PMB 351683, 2301 Vanderbilt Place, Nashville, TN 37232, USA

Corresponding author. E-mail: j.wittig@vanderbilt.edu

James Bentley ${ }^{1}$ and Lawrence F. Allard

Materials Science and Technology Division, Oak Ridge National Laboratory, PO Box 2008, Oak Ridge, TN 37831-6376, USA

E-mails: bentleyj48@gmail.com, allardlfjr@ornl.gov

${ }^{1}$ Present address: Microscopy and Microanalytical Sciences, PO Box 7103, Oak Ridge, TN 37831-7103, USA.

\begin{abstract}
In situ high-resolution electron microscopy was used to reveal information at the atomic level for the disordered-to-ordered phase transformation of equiatomic FePt nanoparticles that can exhibit outstanding magnetic properties after transforming from disordered face-centered-cubic into the tetragonal $\mathrm{L}_{0}$ ordered structure. High-angle annular dark-field imaging in the scanning transmission electron microscope provided sufficient contrast between the Fe and Pt atoms to readily monitor the ordering of the atoms during in situ heating experiments. However, during continuous high-magnification imaging the electron beam influenced the kinetics of the transformation so annealing had to be performed with the electron beam blanked. At $500^{\circ} \mathrm{C}$ where the reaction rate was relatively slow, observation of the transformation mechanisms using this sequential imaging protocol revealed that ordering proceeded from (002) surface facets but was incomplete and multiple-domain particles were formed that contained anti-phase domain boundaries and anti-site defects. At 600 and $700^{\circ} \mathrm{C}$, the limitations of sequential imaging were revealed as a consequence of increased transformation kinetics. Annealing for only $5 \mathrm{~min}$ at $700^{\circ} \mathrm{C}$ produced complete single-domain $\mathrm{L} 1_{0}$ order; such single-domain particles were more spherical in shape with (002) facets. The in situ experiments also provided information concerning nanoparticle sintering, coalescence, and consolidation. Although there was resistance to complete sintering due to the crystallography of $\mathrm{L} 1_{0}$ order, the driving force from the large surface-area-to-volume ratio resulted in considerable nanoparticle coalescence, which would render such FePt nanoparticles unsuitable for use as magnetic recording media. Comparison of
\end{abstract}


the in situ data acquired using the protocol described above with parallel ex situ annealing experiments showed that identical behavior resulted in all cases.

\section{$\underline{\text { Keywords }}$}

STEM, FePt, nanoparticles, in situ annealing, phase transformation, $\mathrm{L}_{0}$ order 


\section{Introduction}

In situ experiments using high-resolution transmission electron microscopy (HRTEM) provide a method to study the behavior of materials at the atomic scale. In situ HRTEM is especially useful for investigation of phase transformations in order to gain understanding of the microstructural changes as a function of time and temperature and to determine their mechanisms and kinetics. There are excellent review articles by Sinclair on this topic that describe the development of using heating holders to observe materials reactions with HRTEM [1,2]. In addition to revealing the atomic mechanism, these in situ experiments can in some cases quantify reaction rates that when analyzed with an Arrhenius relationship allows for activation energy determination [3].

However, care must be taken to ensure that the behavior observed in the TEM is consistent with behavior outside the TEM and, where relevant, that thin TEM specimens behave in the same way as bulk materials. Any influences of surface diffusion and electron beam-specimen interactions need to be understood to determine the applicability of the in situ data. Although, in certain experimental situations, electron beam heating is useful to activate defect motion or change the crystal shape [4,5], correct analysis of phase transformation kinetics requires the elimination of any influence of the electron beam on the reaction rate. In situ results should always be compared to ex situ experiments in order to verify the validity of the in situ results. Nevertheless, with careful experimental technique, in situ HRTEM provides information that is otherwise not available with any other experimental method.

The experiments described in this study concern ferromagnetic FePt nanoparticles. In the $\mathrm{L} 1_{0}$ $(\mathrm{CuAuI})$ ordered state, equiatomic FePt exhibits exceptional magnetic properties with uniaxialmagnetocrystalline anisotropy $\left(\mathrm{K}_{\mathrm{u}}\right)$ greater than $10^{7} \mathrm{ergs} / \mathrm{cm}^{3}$ [6]. However, monodispersed FePt nanoparticles produced by chemical synthesis methods [7] are disordered face-centered cubic (FCC) and require annealing to chemically order into the tetragonal $\mathrm{L}_{0}$ structure. This disorderto-order phase transformation has been studied by using x-ray diffractometry (XRD) to monitor the development of the superlattice peaks with annealing time and temperature [7-10]. Figure 1shows an illustrative example of XRD data from as-synthesized monodispersed FePt nanoparticles (average size $4.9 \pm 0.5 \mathrm{~nm}$ as determined by TEM) and after annealing at $700^{\circ} \mathrm{C}$ for $30 \mathrm{~min}$. Clearly annealing at $700^{\circ} \mathrm{C}$ for $30 \mathrm{~min}$ not only ordered the particles (presence of the (001) and (110) superlattice peaks) and produced a tetragonal unit cell (splitting of the (200) and (002) peaks) but the average particle size also increased, as evidenced by the reduced characteristic peak widths. Although it reveals the overall crystallographic state of the particles, $\mathrm{XRD}$ is limited by being biased with diffraction from the larger particles compared to the contribution from the smaller ones and provides no information concerning the actual mechanisms of the phase transformation. A more complete understanding of this transformation requires electron microscopy to observe the structural changes that occur during the annealing heat treatments. 


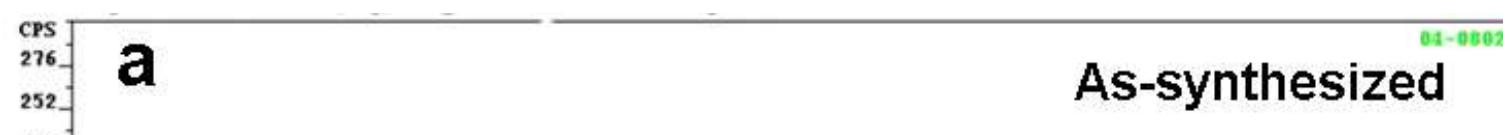

(111)
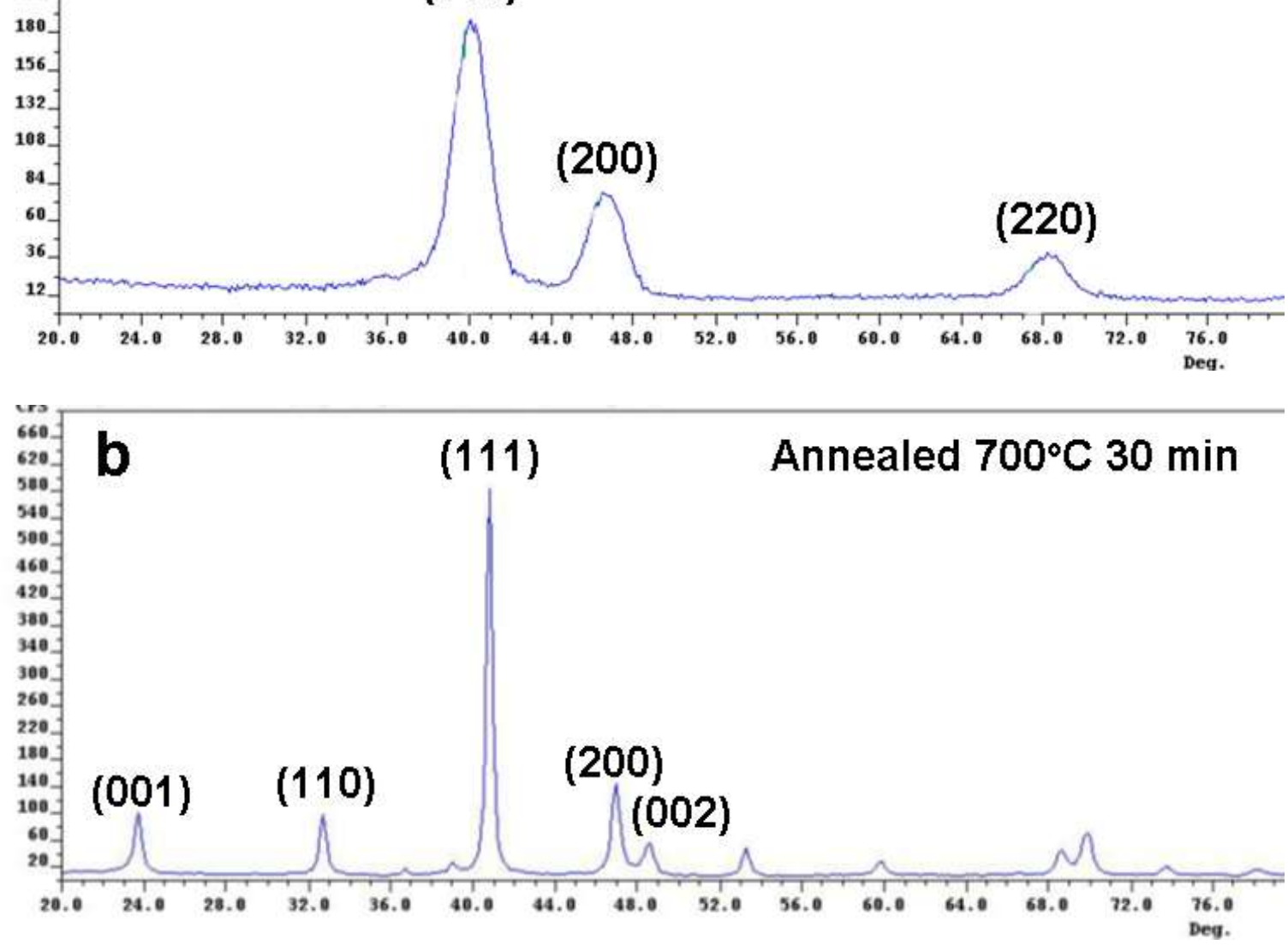

Figure 1. X-ray diffractometer scans of intensity versus $2 \theta$ for (a) the as-synthesized disordered face-centered cubic FePt nanoparticles (b) $\mathrm{L1}_{0}$-ordered FePt nanoparticles after annealing at $700^{\circ} \mathrm{C}$ for $30 \mathrm{~min}$.

In the disordered FCC state, the nanoparticles are superparamagnetic; however, the $\mathrm{L} 1_{0}$-ordered FePt nanoparticles display outstanding magnetic properties [7-8, 11-13] with extremely high $\mathrm{K}_{\mathrm{u}}$ and coercivity that can exceed $22 \mathrm{kOe}$ [8]. Therefore, understanding the $\mathrm{L} 1_{0}$-ordering phase transformation in these FePt nanoparticles is critical for incorporating them into information storage devices that exploit their exceptional magnetic properties. The current study employs in situ heating using a TEM specimen holder with a MEMS-based heating element/specimen support and imaging by high-angle annular dark-field (HAADF) scanning transmission electron microscopy (STEM), also known as atomic-number contrast or Z-STEM, to investigate the development of $\mathrm{L} 1_{0}$ order as well as associated changes in particle shape and particle 
coalescence during annealing. Parts of this work have previously appeared as extended abstracts in conference proceedings [14-19].

\section{Materials and Methods}

The FePt nanoparticles were synthesized using a standard method that is described elsewhere [7]. A surfactant of oleic acid and oleyl amine stabilizes the FePt particle-size distribution with a standard deviation that can be as small as 5\%. One drop of a suspension of FePt nanoparticles in hexane was deposited onto a Protochips "Aduro "TM" disposable 3-mm diameter MEMS device ("E-chip") that serves as both the heating element and the specimen support grid (consisting of a patterned $\mathrm{SiC}$ membrane supporting an electron-transparent holey carbon film and fitted with microfabricated Au electrical contact pads), a custom TEM holder with electrical feed-throughs, and a progammable external stable power supply [20]. The E-chip heater allows specimens to be heated to temperatures up to $1000^{\circ} \mathrm{C}$ in milliseconds through resistance heating by passing a current through the membrane. Each E-chip is calibrated in vacuum, using infrared imaging methods described in [20]. Since the distance from the center of the hole with the carbon film to the edge of the ceramic membrane heater is less than $2 \mu \mathrm{m}$, any thermal gradient would be minimal, maybe a few degrees. As no convenient method exists that can directly measure the temperature of the particles, the assigned $\mathrm{FePt}$ nanoparticle temperature is assumed to be equal to the calibrated ceramic membrane temperature where the uncertainty is on the order of $+/-2$ degrees.

Simultaneous bright-field (BF) and HAADF STEM images were recorded with a sub-0.1-nm probe in a probe-corrected JEOL 2200FS-AC STEM/TEM instrument operated at $200 \mathrm{kV}$. Microscope parameters typically employed include a current of $\sim 30 \mathrm{pA}$ in a probe of nominal diameter $0.07 \mathrm{~nm}$ produced with a convergence semi-angle of $26.5 \mathrm{mrad}$ (MAG mode $8 \mathrm{C}$, aperture 3). Directly calibrated HAADF inner and outer collection semi-angles were 110 and 470 mrad, respectively. Images of 512 x 512 pixels were recorded with $64 \mu \mathrm{s}$ dwell/pixel ( $16 \mathrm{~s}$ total scan time). The difference in atomic number between Fe (26) and Pt (78) allows for high contrast in HAADF STEM images that for suitable crystallographic orientations clearly reveal the chemical ordering. Interestingly, initially contamination during STEM imaging from the presence of the oleic acid and oleyl amine surfactant was severe, was not reduced by the usually effective "beam shower" and even persisted while imaging with the specimen at up to $400^{\circ} \mathrm{C}$. Contamination was only slightly reduced following ex situ annealing in $\mathrm{Ar}-4 \% \mathrm{H}_{2}$ for 30 min at $400^{\circ} \mathrm{C}$ but ceased completely on fresh specimens after in situ annealing at $500^{\circ} \mathrm{C}$ for a short time $(\sim 60 \mathrm{~s})$. Subsequent to in situ annealing, STEM imaging was performed at a slightly elevated temperature of $\sim 150^{\circ} \mathrm{C}$ to eliminate any possibility of new contamination build-up while acquiring STEM images.

After the annealing treatments, the composition of individual FePt nanoparticles studied on the MEMS device was measured by x-ray energy dispersive spectroscopy (EDS) with a Philips CM200 FEG. Initial measurements with a 1-nA STEM probe of <1.5nm FWHM diameter were 
compromised by beam damage, frequently with a dramatic loss of Fe within $\sim 10 \mathrm{~s}$, as revealed by time-series of spectra. Subsequently, to reduce beam damage effects, the current density during EDS analysis was reduced by at least 20-fold with the use of a $\sim 1$-nA nanoprobe manually defocused to just larger than the particle being measured and positioned while viewing the TEM image. Reduced EDS counts (and thus reduced compositional precision) were tolerated with an effective 0.2-0.5 nA on the particle. Even so, confidence was never high that there was no loss of Fe during analyses, just that Fe loss was much reduced from that with a focused probe. Generally, standardless analysis with calculated k-factors, especially those following the early treatment by Zaluzec [21] that use parameterized fits to ionization cross-sections, is convenient and accurate. However, when mixing $\mathrm{L}$ and $\mathrm{K}$ lines, experience has shown that the accuracy is much lower than when using $\mathrm{K}$ lines only. Thus, the EDS data were quantified using an experimental Cliff-Lorimer k-factor ( $\mathrm{Fe} \mathrm{K}_{\alpha}$ versus $\mathrm{Pt}_{\alpha}$ ) obtained from sputtered equiatomic FePt thin films fabricated by Western Digital using their well-characterized standard procedures. Multiple EDS measurements yielded $\mathrm{k}_{\mathrm{PtFe}}=\mathrm{I}_{\mathrm{Fe}} / \mathrm{I}_{\mathrm{Pt}}=0.80 \pm 0.02$ for compositions in at.\% compared to a calculated value of $\sim 1.7$ Early attempts to make Fe-Pt standards from thin films of Fe and Pt sequentially sputter deposited onto rocksalt and subsequently floated off and captured on Be grids were unsuccessful. Cross-section TEM specimens that were produced from depositions onto Si substrates simultaneously with rocksalt, from which the thicknesses of the Fe and $\mathrm{Pt}$ layers were intended to yield the $\mathrm{Fe} / \mathrm{Pt}$ atomic ratio of the standard, indicated that there were problems with the densities of the deposited layers. Typically, EDS data from individual nanoparticles had intensities for $\mathrm{Fe}_{\alpha}$ and $\mathrm{Pt} \mathrm{L}_{\alpha}$ of $\sim 2500$ producing an uncertainty in composition ratio due to counting statistics of $\sim 3 \%$. With additional uncertainties from the measured $\mathrm{k}_{\mathrm{PtFe}}$ and possible Fe depletion due to beam damage, a compositional accuracy of about $\pm 4 \%$ and a precision of about $\pm 3.5 \%$ for comparisons among particles are realistic estimates.

The FePt disorder-to-order transformation was studied at 500,600 , and $700^{\circ} \mathrm{C}$. In addition, the sintering and coalescence behaviors of the FePt nanoparticles were investigated from 500 to $800^{\circ} \mathrm{C}$. These in situ results were compared to ex situ annealing of FePt nanoparticles on grids with a holey carbon film that were annealed in a tube furnace in vacuum.

\section{Results}

\subsection{Electron Beam-Induced Changes}

Figure 2 shows some of the initial results from this study that investigated the influence of the electron beam on the phase transformations. This particular nanoparticle (which develops $\mathrm{L}_{2}$ rather than $\mathrm{L} 1_{0}$ order), initially $\sim 5 \mathrm{~nm}$ in size, changes considerably when exposed to the electron beam at a temperature of only $400^{\circ} \mathrm{C}$, which is below the temperature where ordering occurs outside of the TEM at short time scales, e.g. results from x-ray diffraction [7]. The image in Fig. 2 (a) was acquired after the $1 \mathrm{~min}$ at $500^{\circ} \mathrm{C}$ treatment to remove the surfactant and additional annealing at $400^{\circ} \mathrm{C}$ including imaging for $3 \mathrm{~min}$ at $400^{\circ} \mathrm{C}$. The subsequent images, Fig. 2 (b-h), were acquired at 2 min intervals while continuing to scan with rapid rastering of the 
probe between recording the $\sim 16$ s exposures of $512^{2}$ pixel images. Bright spots in the images around the particle are presumed to be single $\mathrm{Pt}$ atoms on the $\mathrm{C}$ support film. The figure in an earlier published extended abstract is incomplete and the times quoted there are incorrect [16].

Besides the apparently more-or-less continuous slight rotation of the particle that changes the channeling conditions (commonly seen for metallic nanoparticles even at room temperature) the changes in shape are considerable. The surface roughens in Fig. 2 (c) between the weak faceting in Fig. 2 (b) and the more pronounced faceting in Figs. 2 (d-f), and the aspect ratio increases markedly between Figs. 2 (f) and 1 (g). Surprisingly, the image contrast is inconsistent with $\mathrm{L}_{0}$ ordering but instead indicates an $\mathrm{L1}_{2}$-ordered $\mathrm{Pt}_{3} \mathrm{Fe}$ structure. Fig $2(\mathrm{~g})$, especially on the left, exhibits contrast expected for an orientation close to the distinctive $\mathrm{L}_{2}<001>$ projection. Also, assuming only a slight change in particle orientation, the rotation by $45^{\circ}$ and the increase in spacing by $\sqrt{2}$ of the bright-dim superlattice planes between Figs $1 \mathrm{~b}$ and $1 \mathrm{c}$ is difficult to reconcile with the $\mathrm{L} 1_{0}$ structure but is consistent with $\mathrm{L} 1_{2}$. The differences in relevant $\mathrm{d}$-spacings 

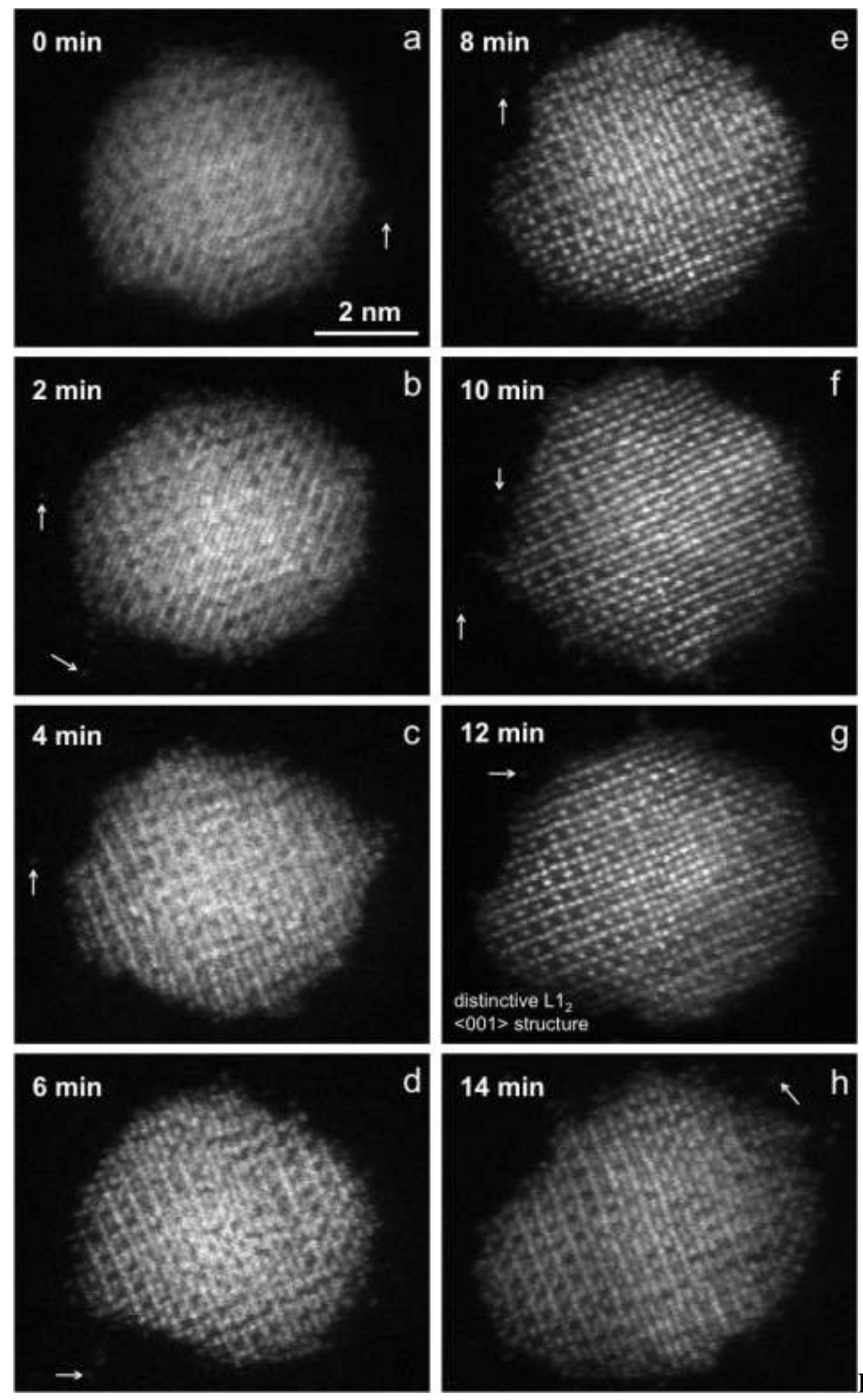

STEM images of an $\mathrm{L1}_{2}$-ordered Fe-Pt nanoparticle at $400^{\circ} \mathrm{C}$ after (a) imaging for $\sim 3$ min and (b-f) at further $\sim 2$ min intervals. Arrows point to examples of bright spots that are presumed to be single Pt atoms on the carbon support film. 
between the structures is $<1 \%$ and thus beyond the accuracy obtainable from these STEM images. The images in Fig. 2 also reveal anti-phase structures, especially but not exclusively between opposite sides of the nanoparticle.

Composition measurements by EDS of many individual nanoparticles indicated a broad distribution with Fe contents ranging from 15 to $>60$ at. $\%$ with a mean of $\sim 30$ at. $\%$.

Undoubtedly, the measured compositions indicate slightly lower-than-actual Fe contents because of beam damage effects that, although much reduced from those experienced with focused probes, are likely not completely eliminated. The nanoparticle synthesis strategy was slightly adjusted to increase the Fe content of the nanoparticles, a change which increased the mean particle diameter from $\sim 5$ to $\sim 6.5 \mathrm{~nm}$.

\subsection{In situ Heating Protocol and Experimental Challenges}

The structural changes and the possible loss of Fe from the nanoparticle in Figure 2 after extended exposure to the electron beam occurred at a relatively low temperature compared to the $\mathrm{L} 1_{0}$ and $\mathrm{L}_{2}$ ordering transformation temperatures outside of the TEM. This is definitive evidence that the electron beam influences the kinetics of the ordering phase transformation. Therefore, all of the following experiments were performed by heating the nanoparticles to $500^{\circ} \mathrm{C}$ for 1-2 min to remove the surfactant, imaging selected clean nanoparticles, annealing at elevated temperatures with the electron beam blanked, and then after each annealing step acquiring images of the same nanoparticles at $\sim 150^{\circ} \mathrm{C}$ to minimize any new specimen contamination, a temperature where no changes to the nanoparticle structure with electron beam exposure have been observed. Care was taken to optimize the imaging conditions away from the nanoparticles being studied after each annealing step (e.g. focus and astigmatism) to minimize the total exposure of the nanoparticle to the electron beam during the successive image acquisitions.

One of the experimental challenges of this research was to locate nanoparticles with a correctly oriented zone axis parallel to the electron beam. Using the BF image to quickly identify strongly diffracting particles was useful, but an additional challenge was that the crystallographic orientation of the nanoparticles would often change over the duration of the sequential annealing treatments. Particles that were properly oriented on a zone axis condition would often wobble off this orientation after the heating cycles. On the other hand, some nanoparticles would actually improve their orientation during the experiment. The best success was for particles that exhibited faceting, where the particle would sit with a low index plane on the carbon support film. For the higher annealing temperatures where the nanoparticles take on a more spherical shape, often the best images could reveal only parallel (002) Pt-rich planes on edge.

\subsection{Annealing at $500^{\circ} \mathrm{C}$}

Annealing near-equiatomic $\mathrm{FePt}$ nanoparticles at $500^{\circ} \mathrm{C}$ for $30 \mathrm{~min}$ produces only minor changes to the disordered FCC powder x-ray diffractometery (XRD) pattern [7]. Although there 
is no evidence for the (200) and (002) peaks splitting, subtle changes in the $2 \theta$ region where the (001) and (110) superlattice peaks develop after annealing at higher temperatures indicate partial $\mathrm{L}_{0}$ ordering. Figure 3 shows FePt nanoparticles ex situ annealed at $500^{\circ} \mathrm{C}$ for $30 \mathrm{~min}$. Although
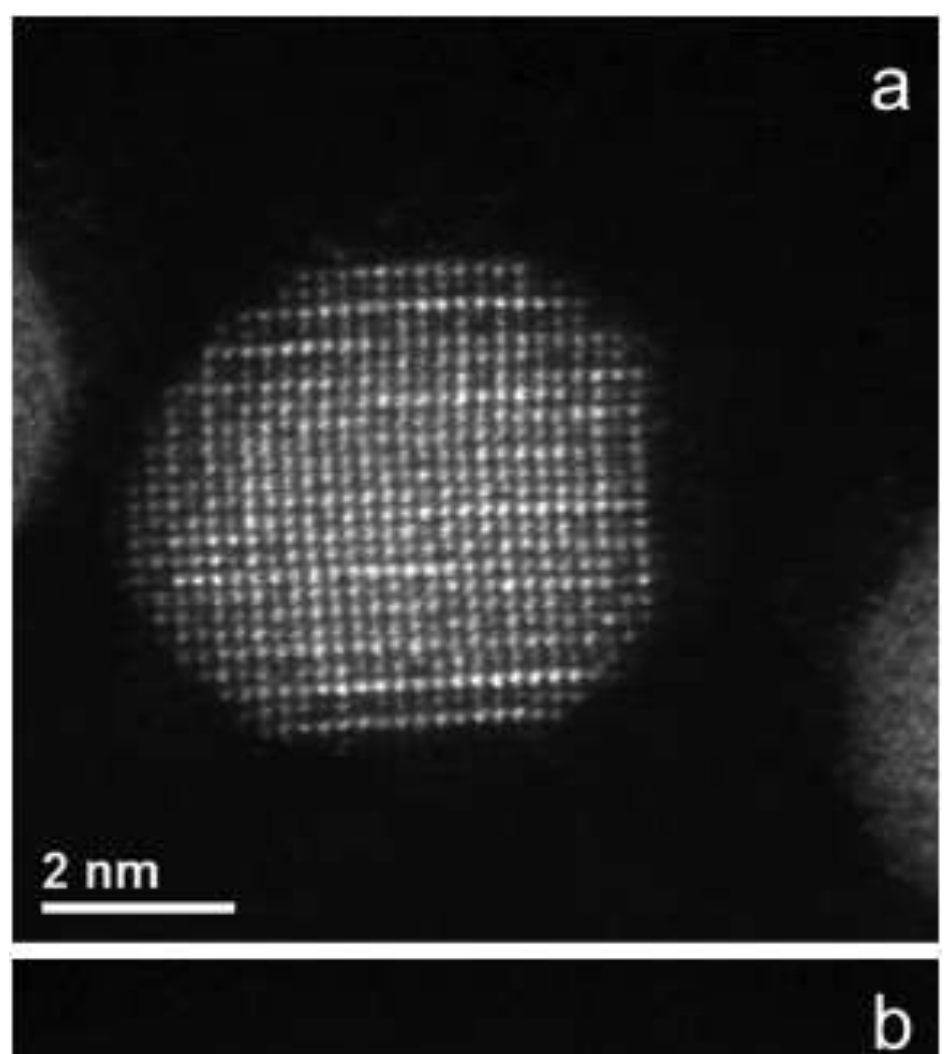

\section{$5 \mathrm{~nm}$}

Figure 3. HAADF STEM images of FePt nanoparticles ex-situ annealed for $30 \mathrm{~min}$ at $500^{\circ} \mathrm{C}$ in vacuum showing (a) incomplete $\mathrm{L} 1_{0}$ order, (b) nanoparticle sintering. 
the HAADF STEM image in Fig. 3 (a) shows (001) facets with enrichment of Pt on the alternating (002) planes, the state of order in the center of the particle cannot easily be discerned. At first sight it might appear that the particle is still highly disordered in the center and thus consistent with the XRD results. However, one would expect that $\mathrm{L} 1_{0}$ ordering similar to that seen near the edge-on (001) facets would also develop for (001) facets normal to the electron beam at the "top and bottom" of the particle. $\mathrm{L} 1_{0}$ order is not obviously revealed for such an orientation and if present may obfuscate the structure in the center of the particle. This effect is of course just another example where atomic structure determination at a single specimen orientation requires a constant projection through the thickness of the specimen to enable simple interpretation. Such a structure is also consistent with the XRD results in that the ordered regions for each variant are only a few unit cells thick and would give rise to significant small particle size broadening of superlattice diffraction peaks. An additional factor, apparent in Fig. 3 (a), is the low degree-of-order in the $\mathrm{L} 1_{0}$ regions, as indicated by significant brightly imaging columns on the darkly imaging "Fe" planes. Such contrast indicates large numbers of anti-site defects. Similar features of the contrast exhibited by the particle in Fig. 3 (a) are also present in the image shown in Fig. 3 (b). The particles in Fig. 3 (b) show that even at $500^{\circ} \mathrm{C}$ there is a driving force for these particles to sinter together, the necked regions also exhibiting characteristic $\mathrm{L1}_{0}$ order. One final observation from Fig. 3 is that careful inspection reveals weak contrast beyond the final brightly imaging Pt plane at the (001) facets. This is clearest for the right and bottom facets in Fig. 3 (a) where such contrast extends out $0.6 \mathrm{~nm}$ or more. Most likely this is Fe-rich material as reported by Prabhudev et al. [22]. The possibility that the Fe is oxidized cannot be excluded. Careful examination of the many images that follow in the present paper reveals that almost without exception there is weak contrast beyond the final brightly imaging Pt plane at (001) facets, often in the form of a single atomic layer. We thus conclude that contrary to first impressions, such facets are not terminated by Pt. This also seems to be the case in the work of Delalande et al [23] which also included in situ annealing of FePt nanoparticles.

A corresponding in situ experiment followed 47 different FePt nanoparticles using the protocol described above for annealing times of $1 \mathrm{~min}, 5 \mathrm{~min}, 15 \mathrm{~min}$, and $30 \mathrm{~min}$ at $500^{\circ} \mathrm{C}$. After the sequential annealing treatment the 47 particles were characterized for composition and size. In a manner similar to previous investigations concerning composition variation in chemically synthesized FePt nanoparticles [24], the nanoparticles studied in this experiment ranged in composition from 37 to 56 at.\% Fe. Figure 4 summarizes these data where the average nanoparticle composition was 47 at. $\% \mathrm{Fe}-53$ at.\% Pt and mean diameter $\left(\left(\mathrm{d}_{\max }+\mathrm{d}_{\min }\right) / 2\right)$ was $6.6 \pm 1.3 \mathrm{~nm}$. Figure 5 is the EDS data from the 6.4-nm diameter particle that is shown in Fig. 6 , which has a composition of 48 at. $\% \mathrm{Fe}-52$ at.\%Pt. After a 1 min anneal at $500^{\circ} \mathrm{C}$ to remove the surfactant, there is already clear evidence for the development of order throughout the particle but perhaps clearest at the top-right of this nanoparticle in Fig. 6 (a). The L1 0 order is noticeably imperfect with many anti-site defects (brightly imaging Pt on what should be planes of $\mathrm{Fe}$ ), in 


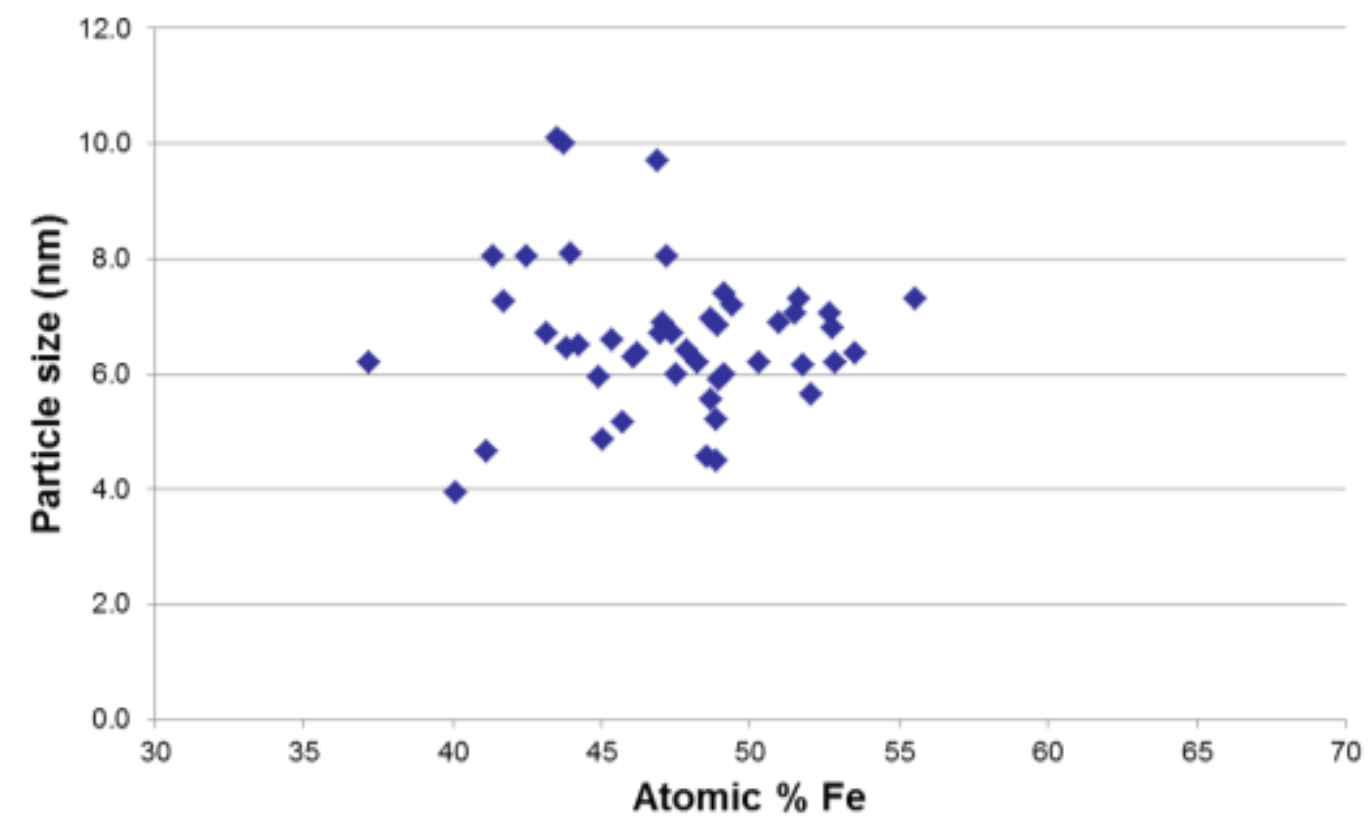

Figure 4. Particle size distribution (average size $=\left(\mathrm{d}_{\max }+\mathrm{d}_{\min }\right) / 2$ ) versus composition for the 47 particles studied for the $500^{\circ} \mathrm{C}$ in-situ annealing experiment.

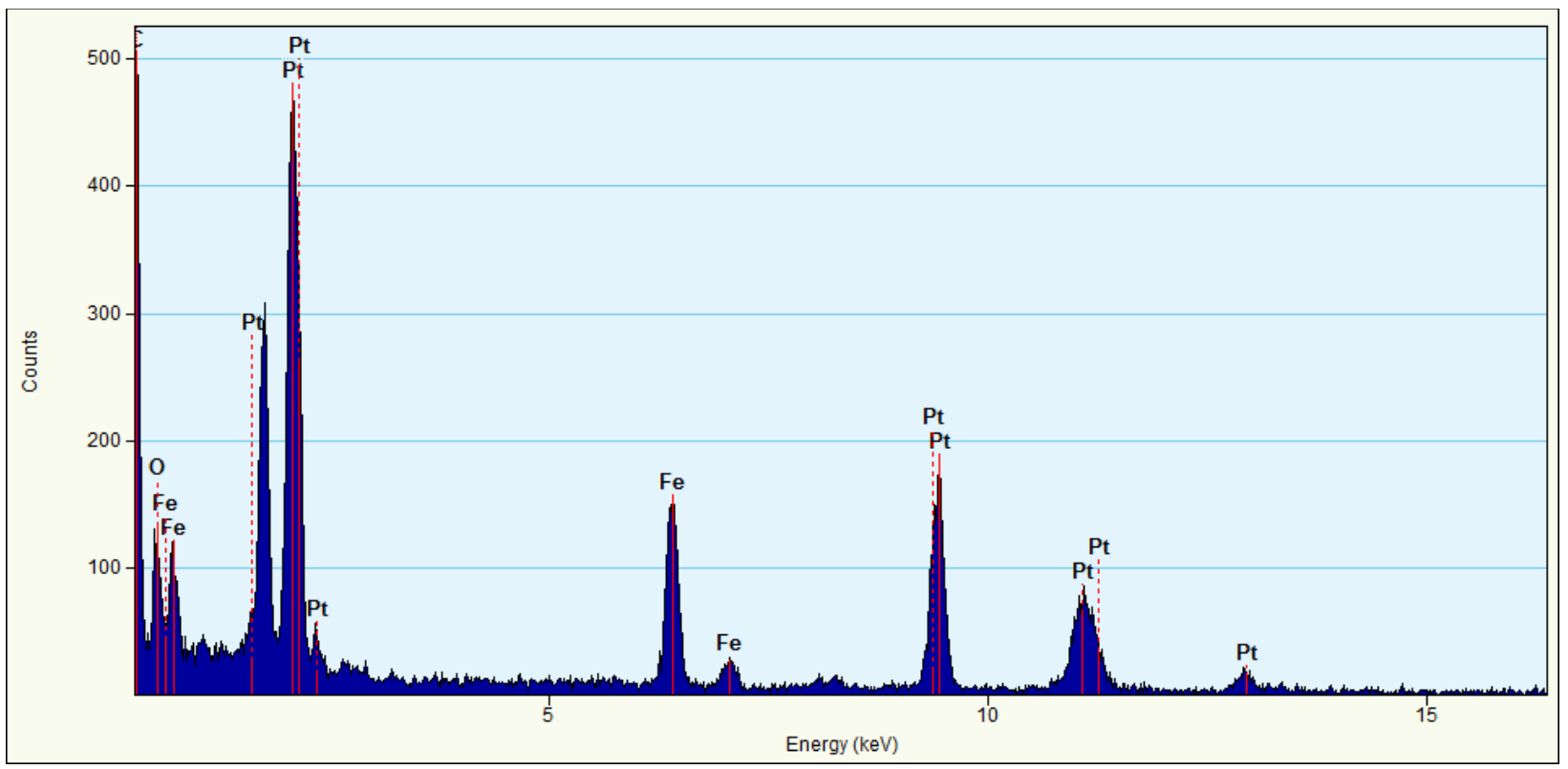

Figure 5. EDS data from the nanoparticle imaged in figure 6 indicating a composition of 48 at. $\%$ $\mathrm{Fe}-52$ at.\% Pt. 

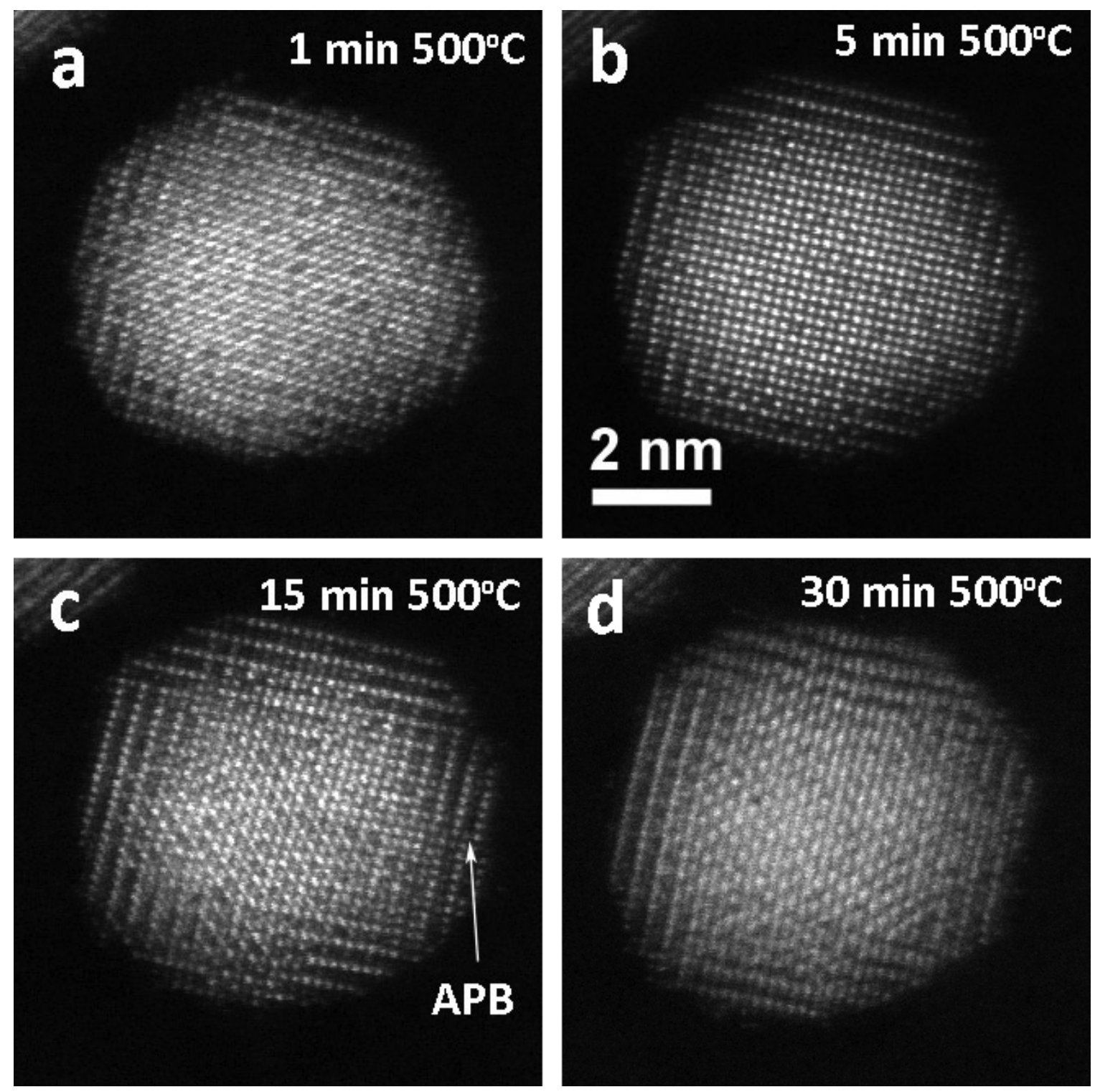

Figure 6. HAADF STEM images from a 6.4-nm diameter FePt nanoparticle with a composition of 48 at. $\% \mathrm{Fe}-52$ at. $\% \mathrm{Pt}$ exhibiting atomic-number contrast from the $\mathrm{L} 1_{0}$-ordered structure after in-situ annealing at $500^{\circ} \mathrm{C}$ for the times indicated.

some cases indicating $\mathrm{Pt}_{3} \mathrm{Fe} \mathrm{L1} 1_{2}$ ordering. The HAADF STEM image in Fig. 6 (b) reveals that after $5 \mathrm{~min}$ of annealing at $500^{\circ} \mathrm{C}$ low-index facets on (100) and (110) are better defined with the c-axis of the tetragonal $\mathrm{L}_{0}$ unit cell forming at two orthogonal orientations on the left side and the top of the particle. The degree of order in these regions has also increased but contrast more indicative of $\mathrm{Pt}_{3} \mathrm{Fe} \mathrm{L1} 1_{2}$ rather than $\mathrm{FePt} \mathrm{L}_{0}$ persists, particularly near the bottom of the image and for the second row of unit cells in from the left-hand (001) facet. It is interesting to compare Fig. 6 (a) and Fig. 2 (a), where some common features are present (such as the small dark 
patches indicating regions of low $\mathrm{Fe} / \mathrm{Pt}$ ) bearing in mind that the particle in Fig 2 (a) had experienced annealing at $400^{\circ} \mathrm{C}$ in addition to the $1 \mathrm{~min}$ at $500^{\circ} \mathrm{C}$ common to both particles.

Fig. 6 (c) shows that annealing for $15 \mathrm{~min}$ at $500^{\circ} \mathrm{C}$ results in the formation of the tetragonal transformation from all four sides of the particle with an anti-phase boundary (APB), where the Pt planes are out of registry, now in the center of a better developed (001) facet on the right-hand side. Careful inspection of the center of the particle reveals traces of the (110) planes resulting from ordering on the top and bottom of the particle with a third orthogonal direction of the caxis. One would, of course, expect that such ordering on the top and bottom surfaces formed concurrently with that on the other sides, and is now revealed as a consequence of a small rotation of the particle with respect to the electron beam direction. In fact, similar but less clearly defined contrast from orthogonal (110) is present in Fig. 6 (a). Impingement of the orthogonal variants of the tetragonal transformation results in a $90^{\circ}$ domain boundary as the transformation continues. Although this particle unfortunately wobbled out of its crystallographic orientation after $30 \mathrm{~min}$ at $500^{\circ} \mathrm{C}$, as shown in Fig. 6(d), the particles in the in situ experiment exhibit the same behavior as those in the ex situ anneal where there is still considerable disorder after annealing for $30 \mathrm{~min}$ at $500^{\circ} \mathrm{C}$.

\subsection{Annealing at $600^{\circ} \mathrm{C}$}

X-ray diffractometry of FePt nanoparticles after annealing at $600^{\circ} \mathrm{C}$ for 30 min indicates that the particles have transformed into the $\mathrm{L} 1_{0}$ superlattice [7]. The (001) and (110) superlattice peaks are clearly present, (002) intensity from the tetragonal unit cell is observed as a shoulder on the (200) peak and the (111) peak is considerably sharper and has a smaller d-spacing than that of the disordered FCC phase. Considerable reduction in the XRD peak widths also indicates that the coherently diffracting regions are larger and thus possibly that nanoparticle coalescence has also occurred. Figure 7 shows HAADF images of particles ex situ annealed at $600^{\circ} \mathrm{C}$ for $30 \mathrm{~min}$ in vacuum. In general, imaging these particles was even more challenging than for particles annealed at $500^{\circ} \mathrm{C}$ owing to less well-defined faceting which failed to promote alignment of the low index crystallographic directions with the electron beam. Figure 7 (a) is a typical HAADF STEM image showing the on-edge (002) Pt planes that indicate a single orientation of $\mathrm{L} 1_{0}$ order throughout most the particle, but with the contrast at the top and at the bottom-left part of this particle suggesting the presence of one or more domain boundaries. The HAADF STEM image in Fig. 7 (b) was the exception among $>75$ recorded images of particles ex situ annealed at $600^{\circ} \mathrm{C}$ in that its low-index crystallographic orientation clearly shows the $\mathrm{L} 1_{0}$ order with multiple domains, domain boundaries and anti-site defects. EDS analysis of the particle in Fig. 7 (b) revealed a slightly $\mathrm{Pt}$ rich composition $\left(54\right.$ at. $\% \mathrm{Pt}-46$ at. $\% \mathrm{Fe}$ ). Annealing for $30 \mathrm{~min}$ at $600^{\circ} \mathrm{C}$ promoted nanoparticle coalescence, as shown in Fig. 7 (c). This particle exhibits what appears to be a grain boundary just to the right of center, but there are $\mathrm{L} 1_{0}(002)$ on each side that are almost parallel, which together with the quite complex arrangement of domain boundaries present in both halves, leads to a conclusion that it may be a domain boundary rather than a grain boundary. 

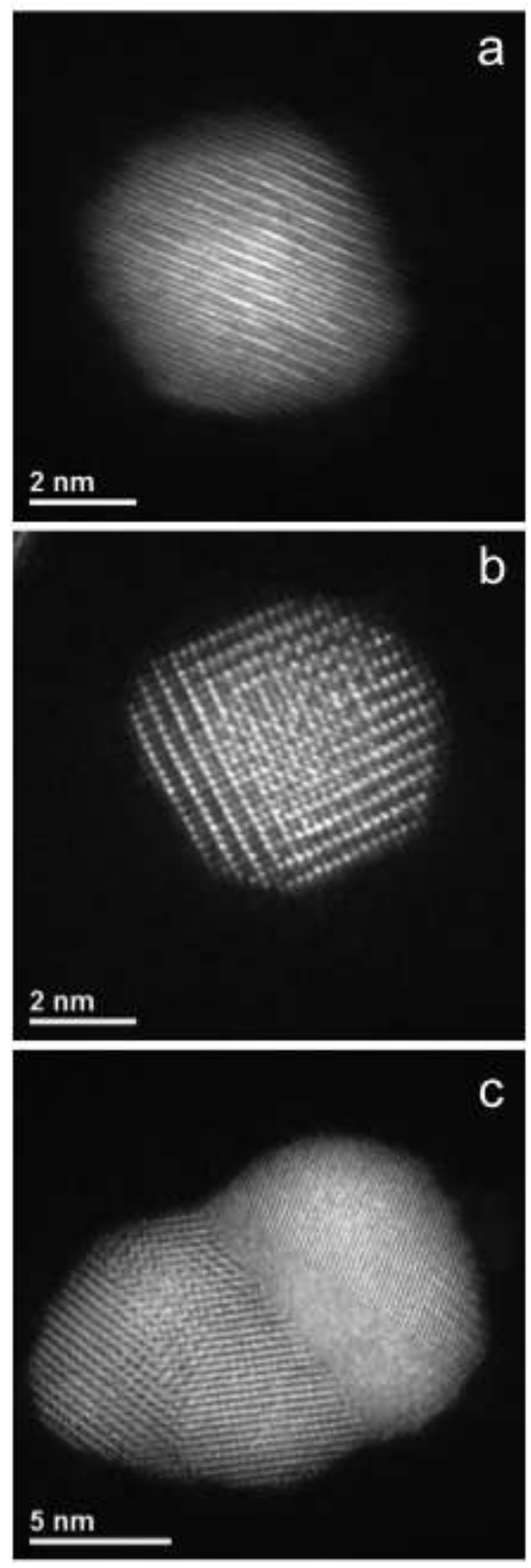

Figure 7. HAADF STEM images of FePt nanoparticles ex-situ annealed at $600^{\circ} \mathrm{C}$ for 30 min. (a) Typical image of an FePt nanoparticle showing ordered planes but not oriented at a low-index zone-axis. (b) Image of an FePt nanoparticle aligned at a low-index crystallographic orientation, an exception from among the $>75$ nanoparticle images recorded. (c) Coalescence of nanoparticles into a polycrystalline particle. 
In situ annealing at $600^{\circ} \mathrm{C}$ produced identical results to those of the ex situ experiment. An example of typical behavior is presented in Fig. 8. Figure 8 (a) is a HAADF STEM image of an
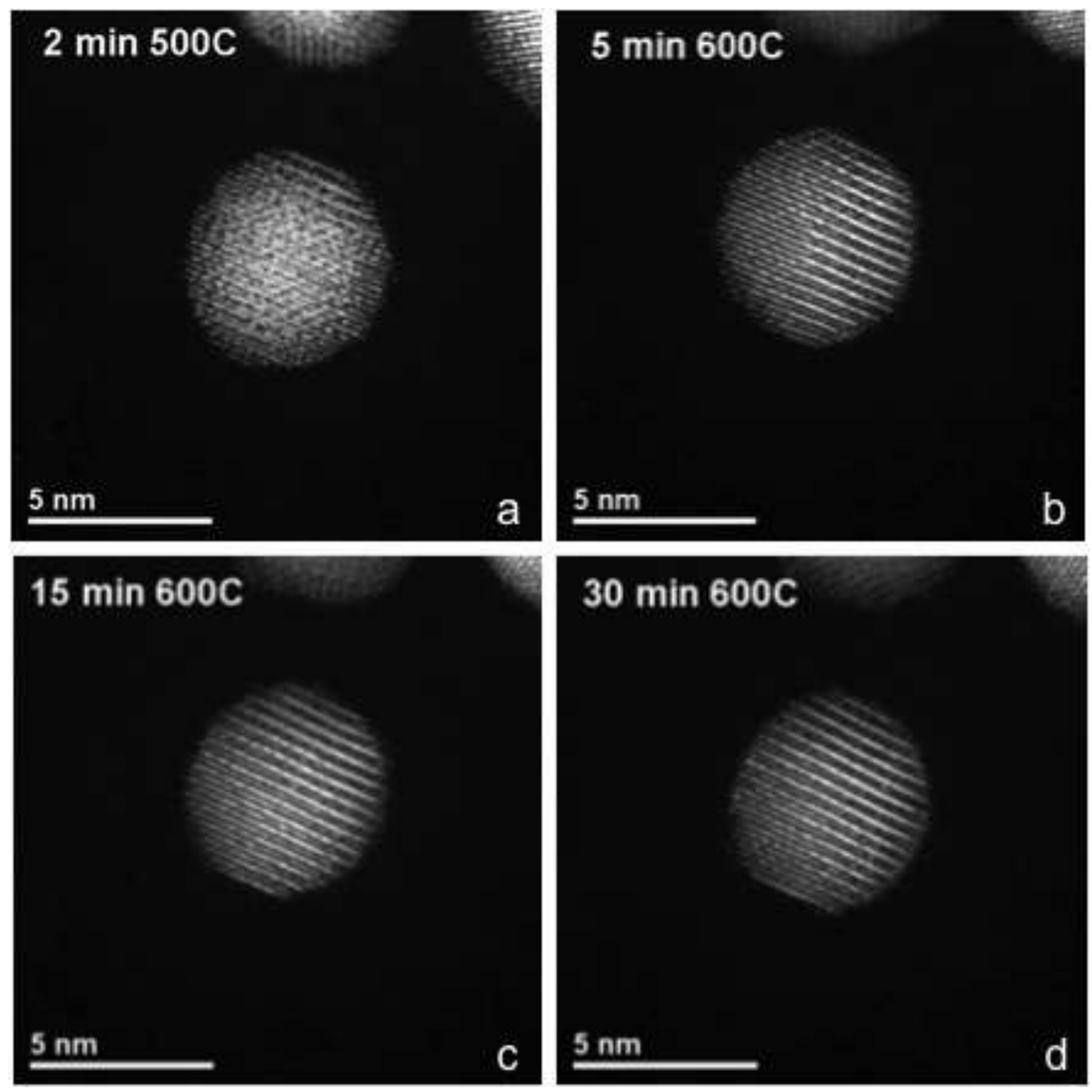

Figure 8 - HAADF STEM images of an FePt nanoparticle after in-situ annealing at $600^{\circ} \mathrm{C}$ for the times indicated.

FePt nanoparticle after the cleaning treatment of $500^{\circ} \mathrm{C}$ for $2 \mathrm{~min}$. After only $5 \mathrm{~min}$ at $600^{\circ} \mathrm{C}$, the particle appears to have transformed into the $\mathrm{L} 1_{0}$-ordered structure with a domain boundary and anti-site defects that hint of weak $\mathrm{L}_{2}$ ordering in some regions. The right side exhibits the characteristic (002) Pt planes on edge whereas the left side has a different c-axis direction and 
(002) plane orientation. The typical lack of faceting after annealing at $600^{\circ} \mathrm{C}$ is also apparent in this example. Continued annealing at $600^{\circ} \mathrm{C}$ for $15 \mathrm{~min}$ (see Fig. 8 (c)) and the full $30 \mathrm{~min}$ (see Fig. 8 (d)) produces only small changes where the ordered domain on the right side grows at the expense of the domain on the left. Consistent with the ex situ results, 30 min of annealing at $600^{\circ} \mathrm{C}$ results in ordered particles with multiple domains and domain boundaries.

\subsection{Annealing at $700^{\circ} \mathrm{C}$}

The HAADF STEM images in Fig. 9 summarize the particle structures produced by ex situ annealing for $30 \mathrm{~min}$ at $700^{\circ} \mathrm{C}$ in vacuum. The nanoparticle shown in Fig. 9 (a) consists of a single $\mathrm{L} 1_{0}$-ordered domain and is non-faceted but not exactly spherical. Nanoparticle coalescence at $700^{\circ} \mathrm{C}$ results in both large, often irregular, and sometimes polycrystalline particles, as shown in Fig. 9 (b) as well as particles that begin to sinter together but do not completely coalesce, as shown in Fig. 9 (c). The in situ results in Fig. 10 reveal the increase of the ordering kinetics at $700^{\circ} \mathrm{C}$. After only $1 \mathrm{~min}$ at $700^{\circ} \mathrm{C}$, the nanoparticle shown in Fig. 10 (b) is completely ordered with a single domain boundary. This domain boundary has disappeared after $5 \mathrm{~min}$ at $700^{\circ} \mathrm{C}$, as revealed in Fig. 10 (c), where the particle is already a single $\mathrm{L} 1_{0}$-ordered domain exhibiting only a weak tendency to facet on (001) and (110). Continued in situ annealing for up to $30 \mathrm{~min}$ at $700^{\circ} \mathrm{C}$ only serves to produce changes in the nanoparticle shape, as shown in Fig. $10(d)$.

\subsection{In situ Sintering and Coalescence}

The in situ coalescence of the nanoparticles was consistent with the observations made from the ex situ experiments; at $500^{\circ} \mathrm{C}$ the particles sinter but do not coalesce, at $600^{\circ} \mathrm{C}$ the particles coalesce into polycrystals, and at $700^{\circ} \mathrm{C}$ the particles coalesce, but on occasion form only sintered necks between particles. The in situ experiments offer some insight into this behavior. Figure 11 (a) shows two nanoparticles after $60 \mathrm{~min}$ at $500^{\circ} \mathrm{C}+30 \mathrm{~min}$ at $600^{\circ} \mathrm{C}$. The $\mathrm{Fe}$ contents measured by EDS were 41 at.\%Fe for the particle on the left and 47 at.\%Fe for the particle on the right. There is clear evidence of $\mathrm{L} 1_{0}$ ordering at the surfaces, and although it seems that the particles are disordered in their centers, it is more likely that they are oriented inappropriately to reveal other variants of $\mathrm{L} 1_{0}$ order. The neck where the sintering is occurring also shows $\mathrm{L}_{0}$ order, but the relative particle orientations result in a grain boundary across the neck. Further annealing for $30 \mathrm{~min}$ at $650^{\circ} \mathrm{C}$ increases the amount of sintering, as shown in Fig. 11 (b), and results in larger $\mathrm{L}_{1}$-ordered domains in the particle on the right. The particle on the left is out of $\mathrm{L}_{0}$ contrast except for a small region adjacent to the grain boundary. The two particles are attempting to coalesce into a single particle although there is still some necking at this interface. 

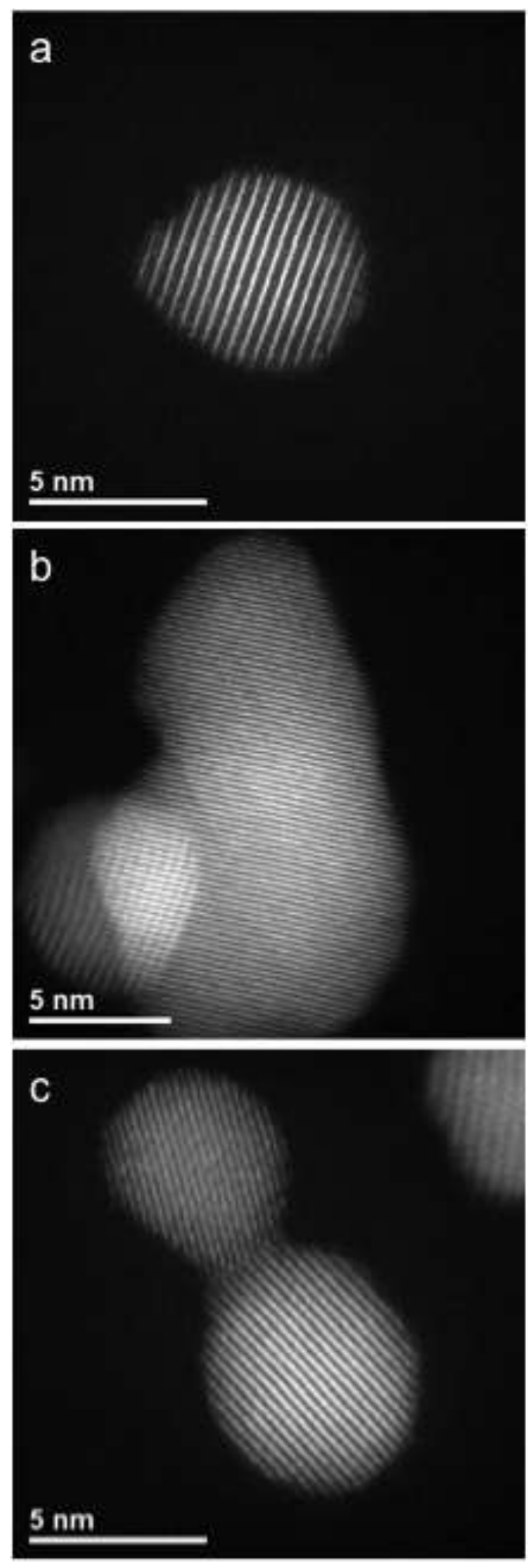

Figure 9. HAADF STEM images of FePt nanoparticles ex-situ annealed at $700^{\circ} \mathrm{C}$ for $30 \mathrm{~min}$. (a) $\mathrm{L1}_{0}$-ordered particle. (b) Coalesced nanoparticles. (c) Sintering but no consolidation of two particles. 


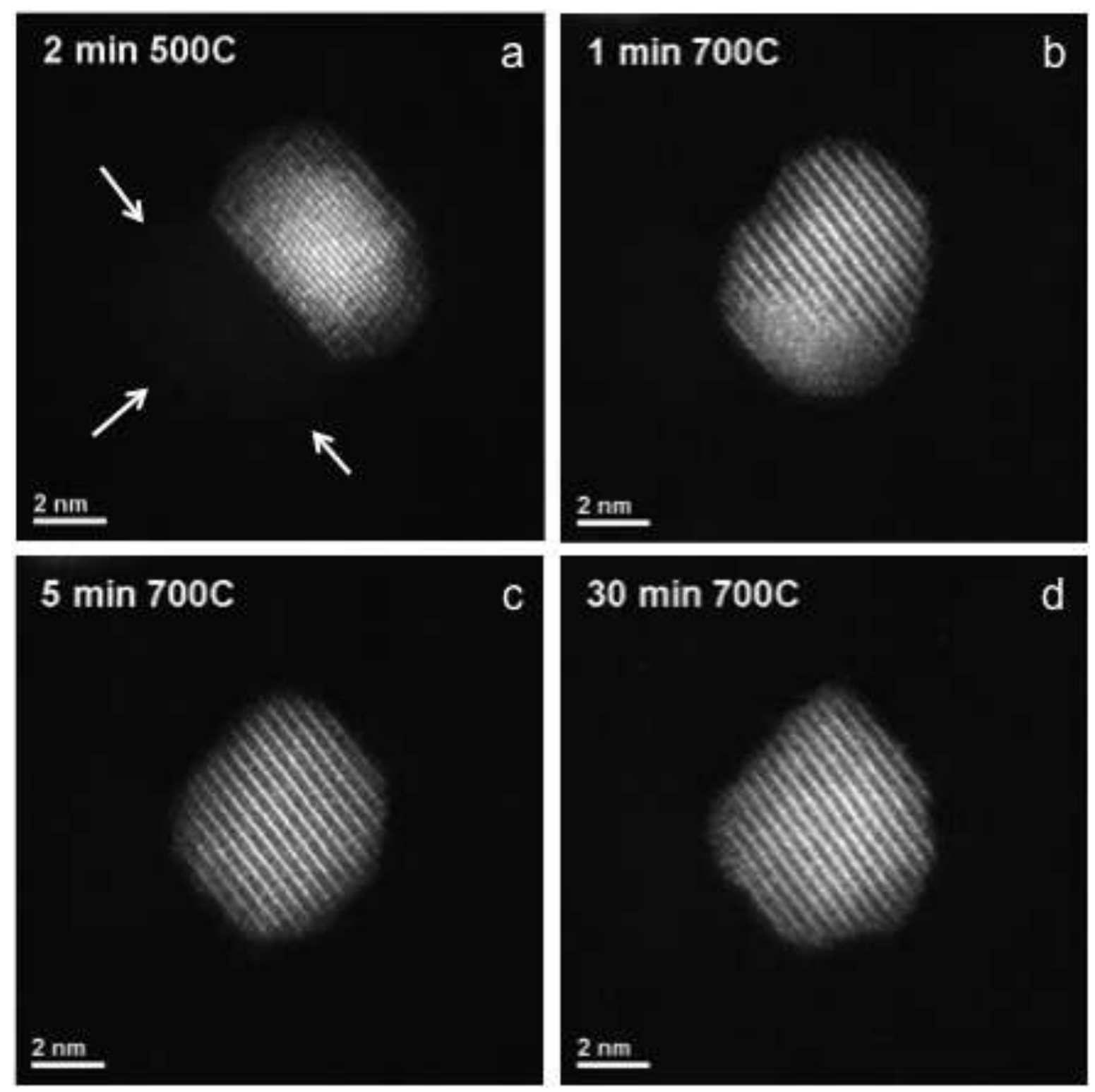

Figure 10. HAADF STEM images of a FePt nanoparticle after in-situ annealing at $700^{\circ} \mathrm{C}$ for the times indicated. Note the "ghost" image arrowed in (a). 

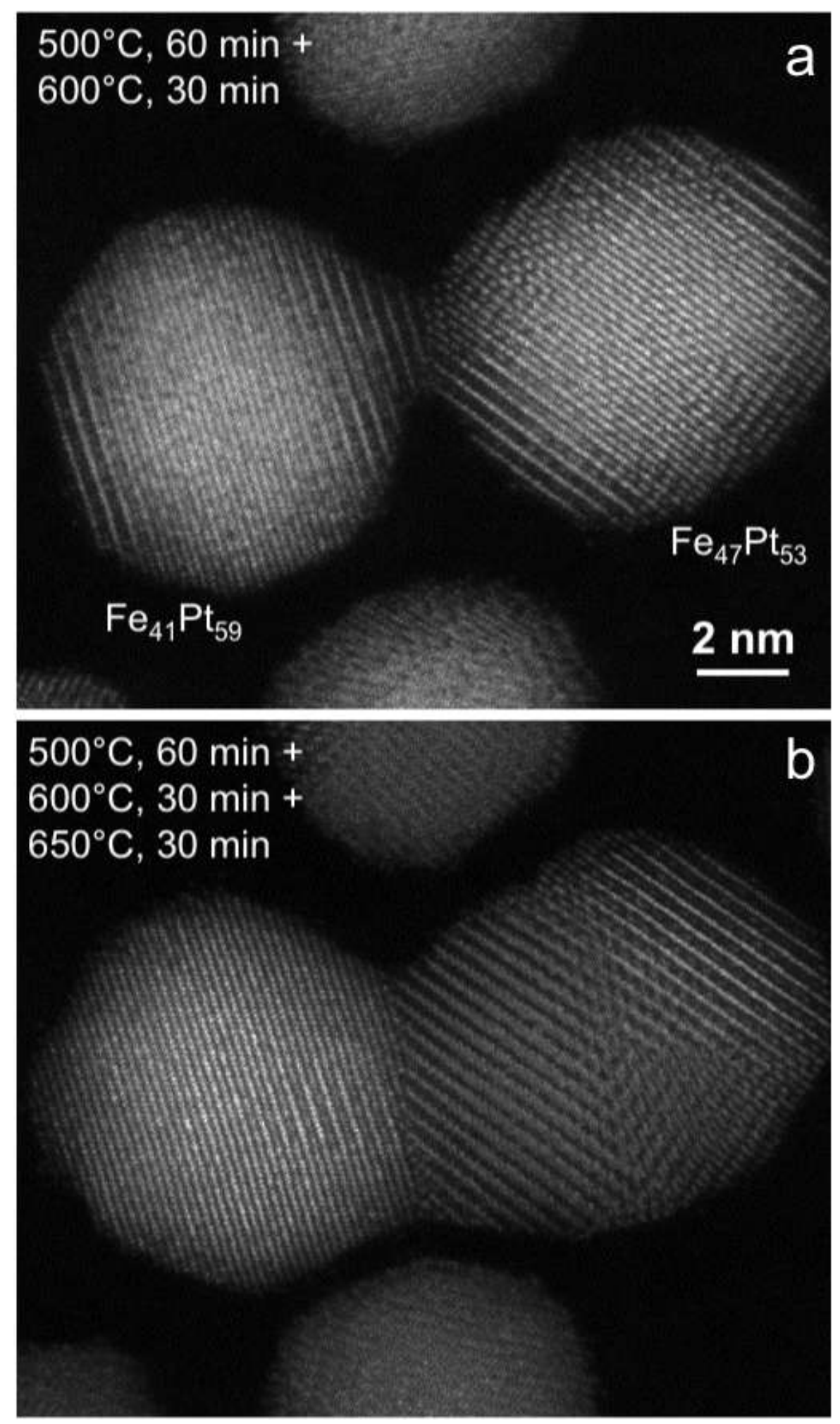

Figure 11. Sintering and coalescence of FePt nanoparticles. (a) After in-situ annealing at $500^{\circ} \mathrm{C}$ for $60 \mathrm{~min}+600^{\circ} \mathrm{C}$ for $30 \mathrm{~min}$. (b) After additional in-situ annealing at $650^{\circ} \mathrm{C}$ for $30 \mathrm{~min}$. 
The lower magnification HAADF STEM images of Fig. 12 show four snapshots from a complex sequence of annealing steps. Initial annealing at $500^{\circ} \mathrm{C}$ for $15 \mathrm{~s}$ is required to remove the organic surfactant from the particle surfaces. However, even after this short time some of the
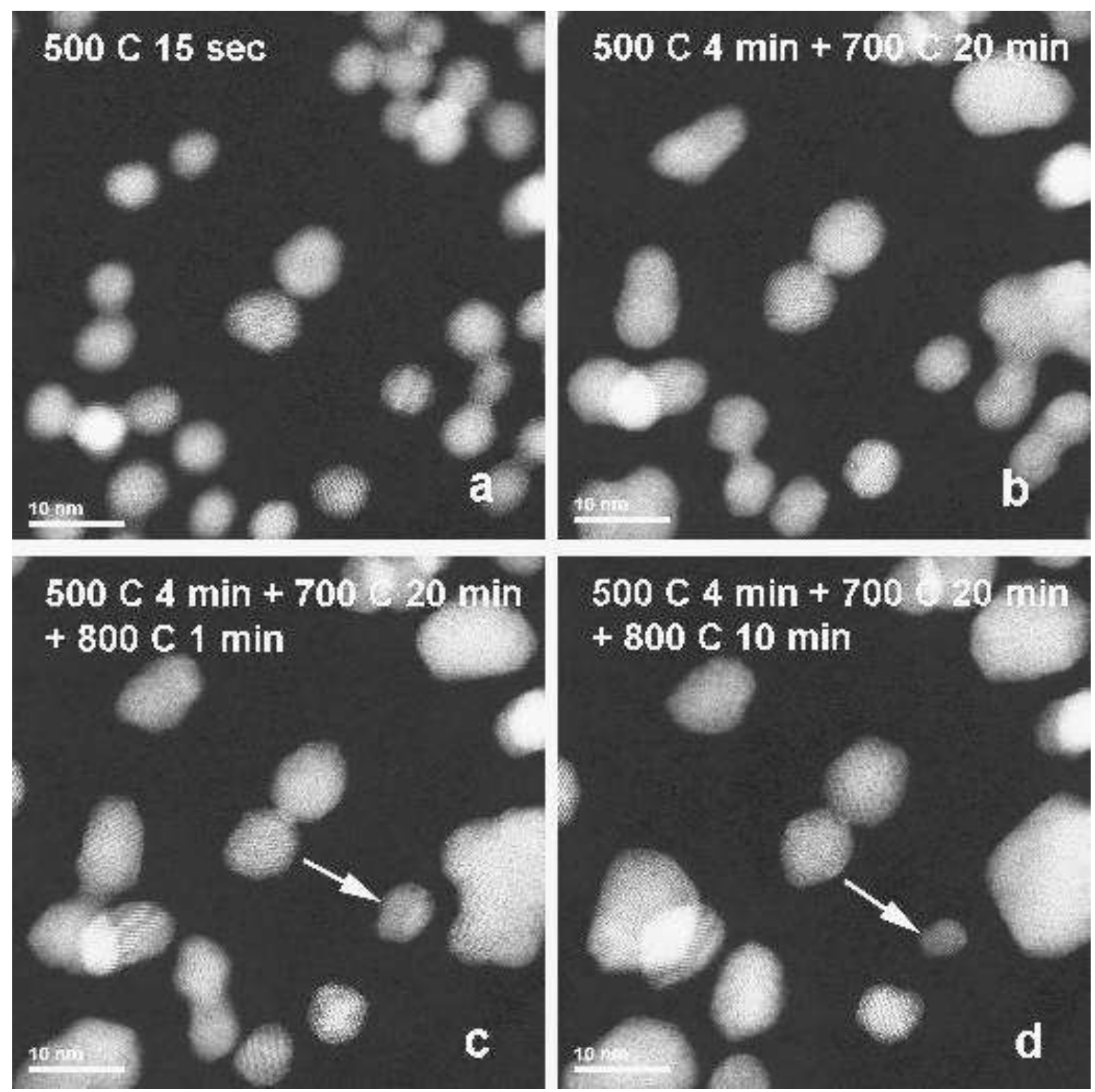

Figure 12. HAADF STEM images showing sintering and coalescence of FePt nanoparticles after in-situ annealing. Evidence of Ostwald ripening is indicated by the arrow in (c-d). See text for details.

nanoparticles have already begun sintering together, as seen in Fig. 12 (a). Continued annealing at $500^{\circ} \mathrm{C}$ for up to $4 \mathrm{~min}$ did not significantly change the particle distribution. Increasing the temperature to $700^{\circ} \mathrm{C}$ increased the rate of sintering and individual nanoparticles coalesced into polycrystalline particles. The $700^{\circ} \mathrm{C}$ annealing was interrupted after six 5-s intervals, three 10 -s 
intervals, two 15 -s intervals, three 30 -s intervals, two 60-s intervals, a 300-s interval and a final $600 \mathrm{~s}$ for a total of 20 minutes at $700^{\circ} \mathrm{C}$, after which the particles appeared as in Fig. 12 (b).

Similarly to the behavior at $500^{\circ} \mathrm{C}$, the majority of the changes to the nanoparticle distribution at $700^{\circ} \mathrm{C}$ occurred in the first few minutes of annealing with little change after extended times. Additional annealing at $800^{\circ} \mathrm{C}$ resulted in dynamic coalescence after the first minute, including coalesced particles becoming more equiaxed, as shown in Fig 12 (c). Continued annealing at $800^{\circ} \mathrm{C}$ resulted in the formation of large single crystal particles and evidence of Ostwald ripening, the latter shown by the arrows in Fig. 12 (c-d). A final interesting observation is that the two particles in the center of the image never fully coalesced (although after many intermediate changes in shape, the size of the upper particle appears to have increased). Closer inspection revealed that these two particles have their $\mathrm{L} 1_{0} \mathrm{c}$-axes at orthogonal orientations. The reduction in surface energy from coalescence is apparently insufficient to offset the energy increase of the larger domain boundary area that would be created by combining these two ordered FePt nanoparticles.

\section{Discussion}

\subsection{Beam-induced changes}

The loss of Fe during EDS analysis and, by extension, probably also during STEM imaging was initially unexpected because $200 \mathrm{kV}$ is well below the (bulk) displacement threshold voltage expected for Fe in FePt via the so called "knock-on" damage from elastic collisions between beam electrons and specimen atoms. Egerton has provided a comprehensive review of beam damage [25]. At surfaces, it is generally accepted that atomic bonding is weaker, and so sputtering of atoms at the electron-exit surface can occur for accelerating voltages well below the bulk displacement threshold voltage. Simple models [25-26] suggest that Fe (at least in unalloyed Fe) can be sputtered at accelerating voltages well below $200 \mathrm{kV}$ and further that sputtering is enhanced for nanoparticles because they have a large fraction of surface atoms at even more-weakly bound sites at surface steps and kinks [25]. Therefore sputtering could be the dominant mechanism responsible for Fe loss. Certainly, beam damage including "hole drilling" at beam energies well below the (bulk) displacement threshold has been a commonly observed effect since soon after the introduction of dedicated FEG-STEMs and, furthermore, is frequently more pronounced in the presence of thin oxide films on the surface of metallic specimens. No doubt this latter effect is related to hole drilling in electrically insulating oxide specimens where the Knotek-Feibelman [27] mechanism was invoked in early explanations of hole drilling [28, 29]. Conventional wisdom is that surface carbon (including contamination) is actually helpful in preventing hole drilling [26], perhaps in the case of contamination as a sacrificial surface species that self-heals by surface diffusion as it is sputtered away. However, in the case of FePt nanoparticles deposited on a carbon support film (carbon at the electron-exit side), we speculate whether, under the action of the electron beam, the carbon might react with the Fe, exacerbating rather than reducing the loss of Fe from FePt. Interestingly, we have observed that annealing such specimens in situ, but without exposure to the beam, at $900^{\circ} \mathrm{C}$ or higher results in rapid and 
complete loss of Fe from the nanoparticles but without any apparent graphitization of the support film or formation of an iron carbide. This effect is completely unexpected from considerations of the equilibrium phase diagram [30], since the disordering and liquidus temperatures are at least $300^{\circ} \mathrm{C}$ and $500^{\circ} \mathrm{C}$, respectively, higher than these elevated annealing temperatures.

In the context of beam-induced changes, the observations presented in Fig. 2 raise many questions and prompt some speculation. Among the leading unanswered questions is why a $\mathrm{Pt}_{3} \mathrm{Fe}$ $\mathrm{L1}_{2}$-ordered structure develops. The nanoparticle could have been $\mathrm{L1}_{2}$-ordered before the observations began, this order perhaps developing during the short anneal at $500^{\circ} \mathrm{C}$ (to remove the surfactants to prevent contamination) rather than during annealing at $400^{\circ} \mathrm{C}$, including the $\sim 3$ min of imaging at $400^{\circ} \mathrm{C}$ before Fig. 2 (a) was recorded. Since the bulk binary Fe-Pt equilibrium phase diagram [30] indicates the $\mathrm{L}_{0}$ and $\mathrm{L}_{2}$ phase fields are broad and biased to lean $\mathrm{Pt}$ compositions with the $\mathrm{Pt}_{3} \mathrm{Fe}$ phase field extending to less than 60 at.\% $\mathrm{Pt}$ at $600^{\circ} \mathrm{C}$, the particle was most likely already sufficiently enriched in $\mathrm{Pt}$ as a consequence of the wider-than-desired spread in composition of the first batch of synthesized nanoparticles. Subsequent syntheses attempted to boost the Fe content at the expense of increasing the mean particle size from $\sim 5$ to $\sim 6.5 \mathrm{~nm}$ diameter. The dark patches in Fig. 2 (b) almost certainly indicate regions of low Pt/Fe and thus imperfect order in $\mathrm{L1}_{2}$-ordered $\mathrm{Pt}_{3} \mathrm{Fe}$, the superlattice planes of which are alternately $\mathrm{Pt}$ and PtFe. Presumably, the $\mathrm{L}_{2}$ structure for Pt contents below the ideal 75 at. $\%$ contains anti-site defects with Fe atoms on some of the Pt-sublattice sites.

Another unanswered question is how the combination of modest temperature and beam stimulation promotes the diffusion necessary to account for the observed structural changes. It is a little difficult to envisage how the lattice vacancies necessary for diffusion-mediated development of long-range order could be produced by surface sputtering, or alternatively how the observed changes could occur by surface diffusion alone. However, the quite dramatic changes in particle shape no doubt predominantly occur by surface diffusion and it is reasonable to expect concomitant ordering as new asperities are formed. Presumably the composition of the nanoparticle was not constant during the observations, but rather that the Fe content continually decreased due to preferential sputtering, if even by only a modest amount compared to the gross effects observed during EDS analyses. It is perhaps worth pointing out that less severe changes might be expected for equivalent atomic-resolution in situ experiments performed in the TEM mode, where carefully controlled electron dose-rates of $\sim 10^{3}$ electrons $/ \AA^{2} / \mathrm{s}$ [31] are much lower than the typical dose rate of $1.2 \times 10^{5}$ electrons $/ \AA^{2} / \mathrm{s}$ averaged over one frame in the present work. However, lower dose rates in the TEM mode may be offset by the continuous imaging frequently employed, such as for video recording. We reiterate that beam-induced changes need to be fully considered when performing in situ studies of phase transformations such as the FCCto- $1_{0}$ transformation in FePt nanoparticles and indeed in related in situ studies such as chemical reactions in gas or liquid cells [31,32].

\subsection{Sequential Acquisition and Orientation Limitations}


There are many examples of real time observation of in situ TEM heating experiments, specifically in thin foils, where the electron beam does not influence the reaction rate [33-35]. In contrast, all in situ STEM images are acquired by sequential acquisition as the scanning time between frames does not capture real time movement of the atoms. For the ordering phase transformation in FePt nanoparticles, the large surface-to-volume ratio of the nanoparticles (> $20 \%$ of the atoms are on the surface for a $5 \mathrm{~nm}$ diameter particle) and the accompanying electron beam-induced changes described above also require that the in situ heating experiments are performed with the electron beam blanked during annealing, which increases the time between observations of the phase transformation. However, the exceptionally high heating and cooling rates $\left(>10^{6} \mathrm{C} / \mathrm{s}\right)$ that are possible with the thin ceramic MEMS heating membrane allows for heating steps of any selectable temperature and time interval. Since there is minimal specimen drift during these heating intervals, the phase transformation of individual nanoparticles can be easily followed.

Although not as satisfying as a continuous movie, the sequential snapshots of the transformation provide valuable information concerning the atomic mechanisms of the ordering phase transformation and nanoparticle coalescence. At intermediate temperatures, such as $500^{\circ} \mathrm{C}$, the phase transformation occurs slowly enough that the development of faceting and the creation of antiphase domain boundaries can be studied by interrupting the experiment at strategic times. For annealing at $500^{\circ} \mathrm{C}$ the results show that the majority of the transformation occurs in the first 10 to 15 minutes, so stopping the experiment after 1,5 , and 15 minutes provides insight into the mechanisms of this phase transformation that are only available using this sequential acquisition in situ heating protocol.

Another experimental limitation encountered in this work concerns the difficulty of discerning the configuration, or even the presence, of differently ordered domains in a single particle that is not oriented with a suitable low-index zone-axis, notably $\langle 100\rangle$, parallel to the electron beam. Several examples have been presented and uncertainties of interpretation already discussed. There is, of course, no such issue with particles not being at a zone axis as long as they are fully ordered in a single domain and a set of ordered planes is imaged. One further example of orientation-limited interpretation is presented in Fig. 13, which shows HAADF STEM images selected from those recorded during a complex sequence of in situ annealing treatments to which the featured particle was subjected. In figure 13 (a), although the orientation is marginal, it is just possible to detect apparent ordering at the top left and bottom right. Interestingly, in Fig. 13 (b) besides this ordering in the caps being much clearer due principally to a slight rotation from the orientation in (a), the zone axis along the upper-right side is near $\langle 100\rangle$ (a square arrangement) whereas the zone axis along the lower left is near $\langle 110\rangle$ (a diamond arrangement). Neither of these regions exhibit any apparent order. In (c) the particle has rotated again and the zone axis orientations are no longer present in the image. In Fig. 13 (d) further changes in the ordered cap regions are evident but the orientation for planar channeling is degrading. The projected particle size increases throughout the sequence; this could be due to coarsening by Oswald ripening or, 
more likely, due to the particle shape and its rotation. The latter conjecture is supported by the ordered caps in Fig. 13 (d) being more laterally offset from each other than they are in the other images. Based on the annealing behavior of other particles at 600 and $700^{\circ} \mathrm{C}$ described above, ordering throughout the particle would be expected after $30 \mathrm{~min}$ at $650^{\circ} \mathrm{C}$, perhaps in the form of multiple domains, but this cannot be confirmed or refuted. Clearly, particle orientation presents a major limitation in attempts to follow the details of the ordering transformation.
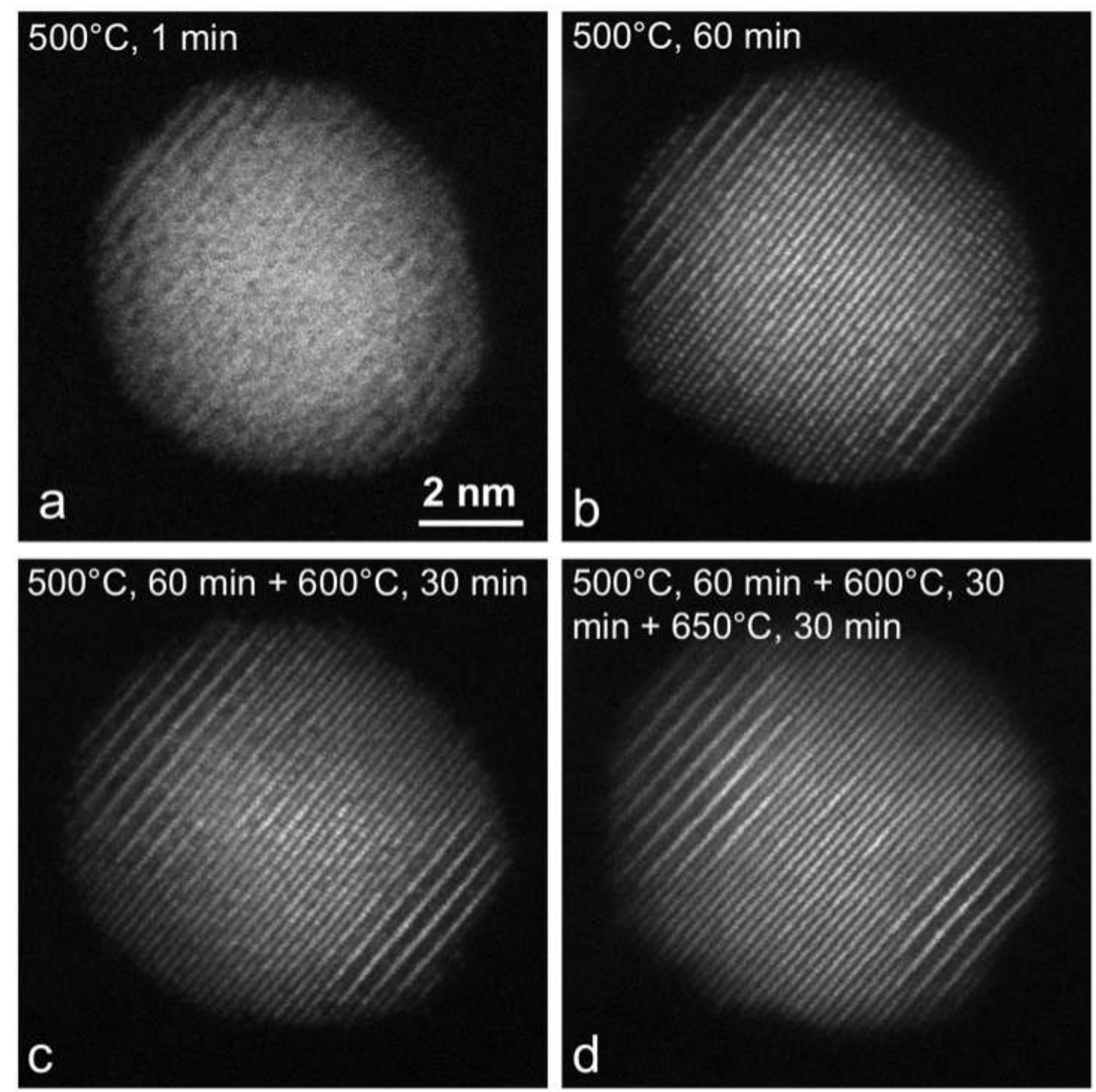

Figure 13. HAADF STEM images of an Fe 52 at.\% - 48 at.\%Pt nanoparticle after in-situ annealing for the indicated times and temperatures. The development of $\mathrm{L} 1_{0}$ order at opposing caps is evident but the particle is not oriented at an appropriate zone-axis to be able to discern the state or order/disorder in the rest of the particle. Note the different zone axis orientations at upper right (near <100>) and lower left (near <110>) in (b). In (c) and (d) the particle has undergone additional rotations. 


\subsection{Ordering Kinetics and Mechanisms}

The sequence of HAADF STEM images from the $500^{\circ} \mathrm{C}$ in situ heating experiment in Fig. 6 , reveals the details from the initial stages of the disordered FCC to ordered $\mathrm{L} 1_{0}$ phase transformation. Faceting of the (002) and (110) planes already begins after the $60 \mathrm{~s}$ at $500^{\circ} \mathrm{C}$ cleaning step. After $5 \mathrm{~min}$ at $500^{\circ} \mathrm{C}$, the $\mathrm{L} 1_{0}$ order starts from the outer layer of atoms by forming alternating $\mathrm{Pt}$ and $\mathrm{Fe}$ rich (002) planes with the center of the particle apparently still highly disordered. Faceting of $\langle 111\rangle$ planes is not observed, in contrast to other ordering phase transformation such as the FCC-to- $\mathrm{L}_{2}$ transformation of $\mathrm{Pt}_{3} \mathrm{Co}$ [36]. The transformation slows down after $15 \mathrm{~min}$ at $500^{\circ} \mathrm{C}$ with ordered domains forming from all six $\{001\}$ facets. The energy of the domain boundaries between the competing facets could be preventing the continued development of the ordered structure at this temperature. The ex situ results also show that $500^{\circ} \mathrm{C}$ is too low to promote complete ordering of these FePt nanoparticles into the $\mathrm{L}_{0}$ structure.

Annealing at $600^{\circ} \mathrm{C}$ increases the kinetics of the ordering phase transformation with apparent reduced facet development, which is most likely the reason for the lack of nanoparticles oriented with [001] parallel to the beam. Of the over 100 nanoparticles imaged after annealing at $600^{\circ} \mathrm{C}$, only the ex situ annealed particle in Fig. 7 (b) exhibited faceting that is similar to that of the particles annealed at $500^{\circ} \mathrm{C}$. Well-developed facets seem to be required in order for the particles to sit on the carbon support film with a low-index zone-axis orientation. The lack of faceting observed in Figs. 8 (c-d) may be related to the lack of a zone-axis condition for the particle orientation. However, the (002) planes terminating on the ends of the particle in Fig. 8 indicate that these facets are indeed present. Both the ex situ and in situ results confirm that the $600^{\circ} \mathrm{C}$ annealing temperature is insufficient to form single domain $\mathrm{L} 1_{0}$ nanoparticles and that domain boundaries are still present after $30 \mathrm{~min}$. Similar to the $500^{\circ} \mathrm{C}$ experiment, the transformation occurs in the first $15 \mathrm{~min}$ at $600^{\circ} \mathrm{C}$ and the structure does not change appreciably with continued annealing.

Increasing the temperature to $700^{\circ} \mathrm{C}$ provides sufficient thermal energy to completely order the FePt particles into the $\mathrm{L}_{0}$ structure. After only 5 minutes at $700^{\circ} \mathrm{C}$, the nanoparticle in Fig. 10 (c) is a single domain $\mathrm{L} 1_{0}$ particle and with the exception of the terminating (002) there is no obvious faceting. Continued annealing served only to change the overall particle shape, where after $30 \mathrm{~min}$ at $700^{\circ} \mathrm{C}$ in Fig. 10 (d) the projected area of the nanoparticle appears to have increased compared to that in Fig. 10 (c). Faceting in the $\mathrm{L} 1_{0}$ nanoparticles at elevated temperatures seems to be limited to the (002) superlattice planes; other low-index facets are presumably a higher-energy configuration than a more spherically shaped surface, the increase in kinks and ledges notwithstanding. 


\subsection{Nanoparticle coalescence}

Coalescence of nanoparticles has been studied theoretically using both molecular dynamic simulations [37,387] and a thermodynamics approach [39]. Most theoretical and experimental studies have concentrated on simple systems such as FCC gold nanoparticles [38-40]. Surface energy, grain boundary energy, and size differences are the main factors that govern sintering, agglomeration, and consolidation. However, when there is a disorder-to-order phase transformation occurring simultaneously, the influences of domain boundary energy and the increased bond energy from like nearest neighbor interactions also need to be considered.

The large surface-area/atom for nanoparticles drives the coalescence behavior. Even at $500^{\circ} \mathrm{C}$ there is a driving force for these FePt particles to sinter together, as shown in Fig. 3 (b).

Interestingly, the orientations of the three left-most parts are almost identical, which seems like too much of a coincidence and indicates that in all probability the constituent particles rotated into alignment as they sintered together. This type of rotation has been observed for $\mathrm{Au}$ nanoparticles using in situ TEM imaging where a 3D rotation allowed the nanoparticles to coalesce without creating high-angle grain boundaries [39]. Such particle rotations probably occurred to avoid the formation of energetically unfavorable grain boundaries in the same way as in the now-classic sphere-on-plate experiments of Herman, Gleiter and Bäro [41]. Further, since the necked regions exhibit characteristic $\mathrm{L}_{0}$ order, the elimination of domain boundaries would be an additional driving force, but there is a $90^{\circ}$ domain boundary at the top of the left-most neck. Surface diffusion is activated at $500^{\circ} \mathrm{C}$, but there is insufficient thermal energy to produce large changes in the nanoparticle shape.

Increasing the annealing temperature to $600^{\circ} \mathrm{C}$, provides the required increase in diffusivity for consolidation into polycrystalline particles. The polycrystalline nanoparticle in Fig. 7 (c), where it appears that at least four individual nanoparticles have been consolidated, indicates that the decrease in total surface energy more than offset the energy of the grain boundaries and domain boundaries created during the coalescence. This type of behavior can also be observed in Fig. 9 (b) after annealing at $700^{\circ} \mathrm{C}$ for $30 \mathrm{~min}$. However, the behavior of the two particles in Fig. 9 (c) shows that other factors are involved and, specifically, that coalescence of $\mathrm{L} 1_{0}$-ordered particles can be energetically unfavorable, owing to the high grain-boundary energy of long-range ordered crystals. An additional factor for $\mathrm{L} 1_{0}$-ordered $\mathrm{FePt}$ is the crystallographic tetragonality that complicates the formation of low-energy grain boundaries. The incomplete sintering behavior is also exhibited in Fig. 11 where, even after annealing for $30 \mathrm{~min}$ at $600^{\circ} \mathrm{C}$ and $30 \mathrm{~min}$ at $650^{\circ} \mathrm{C}$, the two particles have not coalesced but retain a necked region.

The higher energy of grain boundaries between ordered crystals compared with the equivalent grain boundary in disordered material was illustrated for a Rene 95 Ni-base superalloy [42, 43]. At a grain boundary between impinging large $\mathrm{L1}_{2}$-ordered $\mathrm{Ni}_{3}(\mathrm{Al}, \mathrm{Ti}) \gamma^{\prime}$ particles, a thin $(\sim 1 \mathrm{~nm})$ layer of solid-solution $\gamma$ matrix was retained, as revealed by $\mathrm{Cr}$ enrichment and $\mathrm{Ni}$ depletion in 
EFTEM elemental maps ( $\mathrm{Cr}$ partitions strongly to the matrix). The extra energy required to eliminate the grain-boundary layer was thus at least twice the $\gamma-\gamma^{\prime}$ interfacial energy.

The extreme case of this resistance to consolidation is shown in Fig. 12, where the two particles in the center of the image failed to coalesce even after annealing at $800^{\circ} \mathrm{C}$. Higher resolution images show that each particle is an $\mathrm{L}_{1}$-ordered single domain and that the respective c-axes are at $90^{\circ}$ to each other. A $<100>90^{\circ}$ symmetric tilt boundary is a special low-energy boundary for tetragonal crystals but for $\mathrm{L}_{0}$-ordered FePt such a misorientation results in the formation of a domain boundary which would mitigate the reduction of surface energy during consolidation. Consistent with other studies of FePt nanoparticle coarsening [44], in addition to sintering and coalescence which is the dominant mechanism, there are examples of Ostwald ripening such as in Fig. 12 (c-d), where smaller particles are shrinking and nearby larger particles are growing. Presumably the transfer of Fe and Pt atoms from the shrinking particles occurs by surface diffusion across the supporting carbon film.

\section{Summary and Conclusions}

Although in situ high-resolution electron microscopy studies can provide information about the mechanisms and kinetics of phase transformations at the atomic scale, it is critical to consider any effects of the electron beam on the transformation. For the FePt nanoparticles in this study, beam-induced changes at elevated temperatures required annealing to be performed with the electron beam blanked. Comparison of the in-situ results with those from parallel ex situ experiments verified the reliability of the in situ data.

Sequential annealing at $500^{\circ} \mathrm{C}$ revealed the mechanisms of the $\mathrm{L}_{0}$-ordering phase transformation, where initial stages of near-surface ordering produced faceting on (002) and $\{110\}$. The HAADF STEM images suggest that annealing in vacuum creates an outer layer of Fe atoms. There is insufficient thermal energy at 500 or $600^{\circ} \mathrm{C}$ to completely order the nanoparticles into single ordered domains and domain boundaries are observed in multipledomain particles. To produce single-domain ordered nanoparticles required annealing at $700^{\circ} \mathrm{C}$ where the ordering transformation occurs so rapidly that the initial stages cannot be observed. The single-domain ordered particles appear more spherical in shape with only (002) facets remaining. Since it was experimentally challenging to image these nanoparticles at a low-index crystallographic zone-axis condition, the detailed shape of the nanoparticles remains somewhat ambiguous.

Annealing at $500^{\circ} \mathrm{C}$ was sufficient to allow nanoparticle sintering, including observed examples where the nanoparticles had rotated to align the low-index planes in the ordered necked regions. Annealing at higher temperatures resulted in nanoparticle coalescence and consolidation. Rapid nanoparticle consolidation into polycrystalline particles occurs at $600^{\circ} \mathrm{C}$. Higher annealing temperatures yield irregularly shaped particles and, in addition, revealed some limited evidence 
of Ostwald ripening. FePt nanoparticles in the fully ordered condition are resistant to consolidation, owing to the larger increase in energy from forming a high-energy grain boundary compared to any reduction in surface energy from coalescence. To exploit attractive magnetic properties, e.g. in technological applications of improved magnetic recording media, it is important to produce fully $\mathrm{L}_{1}$-ordered single-domain FePt particles of the desired size. Clearly, annealing at too low or too high a temperature fails to produce particles that meet this requirement. Too low and the ordering is incomplete; too high and particle coalescence can dominate. Both are undesirable.

\section{Acknowledgements}

It is a privilege and a pleasure to dedicate this paper to our friends and colleagues, Robert Sinclair and Nestor Zaluzec who have inspired us in our efforts to employ in-situ and analytical electron microscopy for the advancement of materials science and engineering. One of us (James Wittig) acknowledges Bob Sinclair's mentorship beginning with his role as Ph.D. advisor. Jim Bentley and Larry Allard would like to thank Nestor for many years of friendship and helpful advice starting with Nestor's time as a Wigner Fellow at ORNL.

Research partially performed at the former Shared Research Equipment (ShaRE) User Facility at Oak Ridge National Laboratory was supported by the Division of Scientific User Facilities, Office of Basic Energy Sciences, U.S. Department of Energy. The contribution of Prof. C. Lukehart and his group in the Vanderbilt University Chemistry Department for synthesizing the FePt nanoparticles used in this study is gratefully acknowledged, as is Dr. Gerardo Bertero of Western Digital for supplying sputter-deposited thin-film FePt specimens and Dr. Nestor Zaluzec of Argonne National Laboratory for help with calculated EDS k-factors. The ex situ annealing was performed with the assistance of Prof. G.B. Thompson at the University of Alabama Tuscaloosa. JB acknowledges his appointment as Adjoint Professor of Materials Science at Vanderbilt University in contributing to this work.

\section{References}

[1] R. Sinclair, T. Yamashita, M.A. Parker, K.B. Kim, K. Holloway and A.F. Schwartzman, The Development of In Situ High Resolution Electron Microscopy, Acta Crystallogr. Sec. A 44 (1988) 965-975.

[2] R. Sinclair, In Situ High-Resolution Transmission Electron Microscopy of Materials Reactions, Mat. Res. Soc. Bull. 38 (2013) 1065-1071.

[3] T.J.Konno and R. Sinclair, Metal-Mediated Crystallization of Amorphous Germanium in Germanium-Silver Layered Systems, Philos. Mag. B 71 (1995) 179-199. 
[4] R. Sinclair, T. Yamashita and F.A. Ponce, Atomic Motion on the Surface of a Cadmium Telluride Single Crystal, Nature 290 (1981) 386-388 DOI: 10.1038/290386a0.

[5] R. Sinclair, F.A. Ponce, T.Yamashita, D. J. Smith, R.A. Camps, L.A. Freeman, S.J. Erasmus, W.C. Nixon, K.C.A. Smith and C.J.D.Catto, Dynamic Observation of Defect Annealing in CdTe at Lattice Resolution, Nature 298 (1982) 127-131 DOI: 10.1038/298127a0.

[6] K. Inomata, T. Sawa. S. Hashimoto, Effectoflarge boron additions to magnetically hard Fe-Pt alloys, J. Appl. Phys. 64 (1988) 2537-2540 DOI: 10.1063/1.341638.

[7] S. Sun, C.B. Murray, D. Weller, L. Folks, A. Moser, Monodisperse FePt Nanoparticles and Ferromagnetic FePt Nanocrystal Superlattices, Science 287 (2000) 1989-1992 DOI:

10.1126/science.287.5460.1989.

[8] R.D. Rutledge, W.H. Morris, III, M. S. Wellons, Z. Gai, J. Shen, J. Bentley, J.E. Wittig, C.M. Lukehart, Formation of FePt Nanoparticles Having High Coercivity, J. Am. Chem. Soc. 128 (2006) 14210-14211 DOI: 10.1021/ja0633868.

[9] O. Robach, C. Quiros, S.M. Valvidares, C.J. Walker, S. Ferrer, Structure and Pt magnetism of FePt nanoparticles investigated with X-ray diffraction, J. Magnetism and Magnetic Materials 264 (2003) 202-208 DOI: 10.1016/S0304-8853(03)00205-1.

[10] S. Anders, M.F. Toney, T. Thomson, J.-U. Thiele, B.D. Terris, S. Sun, C.B. Murray, X-ray studies of magnetic nanoparticle assemblies, J. Appl. Phys. 93 (2003) 7343-7345 DOI: $10.1063 / 1.1543864$.

[11] B. Stahl, N.S. Gajbhiye, G. Wilde, D. Kramer, J. Ellrich, M. Ghafari, H. Hahn, H. Gleiter, J. Weißmüller, R. Würschum, P. Schlossmacher, Electronic and Magnetic Properties of Monodispersed FePt Nanoparticles, Advanced Materials 14 (2002) 24-27 DOI: 10.1002/15214095(20020104)14:1<24::AID-ADMA24>3.0.CO;2-0.

[12] Y. Ding, S. Yamamuro, D. Farrell, S.A. Majetich, Phase transformation and magnetic moment in FePt nanoparticles, J. Appl. Phys. 93 (2003) 7411-7413 DOI: 10.1063/1.1544495.

[13] S.S. Kang, D.E. Nikles, J.W. Harrell, Synthesis, chemical ordering, and magnetic properties of self-assembled FePt-Ag nanoparticles, J. Appl. Phys. 93 (2003) 7178-7180 DOI: 10.1063/1.1543859.

[14] J.E. Wittig, J. Bentley, A. Lupini, and C.M. Lukehart, Z-STEM of L1 $1_{0}$ Ordering in FePt Magnetic Nanoparticles, Microsc. Microanal. 13[suppl.2] (2007) 1196-7 DOI:

10.1017/S143192760707376X

[15] J.E. Wittig, J. Bentley, L.F. Allard, M.S. Wellons and C.M. Lukehart, n-situ Z-STEM Imaging of Chemical Ordering in FePt Magnetic Nanoparticles, Microsc.. Microanal. 14[suppl 2] (2008) 216-7 DOI: 10.1017/S1431927608087400 
[16] J.E. Wittig, J. Bentley, L.F. Allard, M.S. Wellons and C.M. Lukehart, Beam-induced Changes of FePt Nanoparticles during STEM In-situ Annealing, Microsc. Microanal. 14[suppl.2] (2008) 348-9 DOI: 10.1017/S1431927608087527.

[17] J.E. Wittig, J. Bentley, L.F. Allard, and C.M. Lukehart, Evolution of FePt Nanoparticle Shape and $\mathrm{L1}_{0}$ Order during In-situ Annealing, Microsc.. Microanal. 16[suppl 2] (2010) 1754-5 DOI:10.1017/S1431927610057880

[18] J.E. Wittig, J. Bentley and L.F. Allard, Characterization of FePt Magnetic Nanostructures Using Aberration-Corrected Z-STEM Imaging, Proc. $17^{\text {th }}$ Intern. Microscopy Cong. (eds. G. Solórzano \& W. de Souza) Rio de Janeiro, Brazil, 19-24 Sep. 2010, pp. M12.8

[19] J.E. Wittig and L.F. Allard, Sintering and Coalescence Behavior of L1 $1_{0}$ FePt Magnetic Nanoparticles, Microsc.. Microanal. 18[suppl 2] (2012) 304-5 DOI: 10.1017/S1431927612003376

[20] L.F. Allard, W.C. Bigelow, M. José-Yacamán, D.P. Nackashi, J. Damiano and S.E. Mick, A New MEMS-Based System for Ultra-High-Resolution Imaging at Elevated Temperatures, Microsc. Res. Techniq. 72 (2009) 208-15 DOI 10.1002/jemt.20673.

[21] N.J. Zaluzec, Chapter 4 in: J.J. Hren, J.I. Goldstein and D.C. Joy (Eds.), Introduction to Analytical Electron Microscopy, Plenum Press, New York, 1979.

[22] S. Prabhudev, M. Bugnet, G.-Z. Zhu, C. Bock and G.A. Botton, Surface Segregation of Fe in Pt-Fe Alloy Nanoparticles: Its Precedence and Effect on the Ordered-Phase Evolution during Thermal Annealing, Chem. Cat. Chem. 7 (2015) 3655-64 DOI: 10.1002/cctc.201500380

[23] M. Delalande, M.J.-F. Guinel, L.F. Allard, A.Delattre,R. Le Bris, Y. Samson, P.BayleGuillemaud and P.Reiss, L10 Ordering of Ultrasmall FePt Nanoparticles Revealed by TEM In Situ Annealing, J Phys. Chem. C 116 (2012) 6866-72 DOI: 10.1021/jp300037r

[24] C. Srivastava, D.E. Nikles and G.B. Thompson, Compositional eveolution during the synthesis of FePt nanoparticles, J Appl. Phys. 104 (2008) 064315 DOI: 10.1063/1.2980042.

[25] R.F. Egerton, P. Li and M. Malac, Radiation damage in the TEM and SEM, Micron 35 (2004) 399-409 DOI: 10.1016/j.micron.2004.02.003.

[26] R.F. Egerton, R. McLeod, F. Wang and M. Malac, Basic questions related to electroninduced sputtering in the TEM, Ultramicroscopy 110 (2010) 991-7 DOI:

10.1016/j.ultramic.2009.11.003.

[27] M.L. Knotek and P.J. Feibelman, Ion Desorption by Core-Hole Auger Decay, Phys. Rev. Lett. 40 (1978) 964-7.

[28] I.G. Salisbury, R.S. Timsit, S.D. Berger and C.J. Humphreys, Nanometer scale electron beam lithography in inorganic materials, Appl. Phys. Lett. 45 (1984) 1289-91 DOI:

$10.1063 / 1.95115$ 
[29] S.D. Berger, I.G. Salisbury, R.H. Milne, D. Imeson and C.J. Humphreys, Electron energyloss spectroscopy studies of nanometre-scale structures in alumina produced by intense electronbeam irradiation, Philos. Mag. 55 (1987) 341-58 DOI:10.1080/13642818708208619

[30] O. Kubaschewski, pp 91-94 in: Iron - Binary Phase Diagrams, Springer-Verlag, 1982.

[31] A.L. Koh, E. Gidcumb, O. Zhou and R. Sinclair, Oxidation of Carbon Nanotubes in an Ionizing Environment, Nano Lett., 16 (2016) 856-863 DOI: 10.1021/acs.nanolett.5b03035.

[32] S.C. Lee, J.D. Benck, C. Tsai, J. Park, A.L. Koh, F.Abild-Pedersen, T.F. Jaramillo and R. Sinclair, Chemical and Phase Evolution of Amorphous Molybdenum Sulfide Catalysts for Electrochemical Hydrogen Production, ACS Nano 10 (2016) 624-32 DOI:

10.1021/acsnano.5b05652

[33] R. Sinclair and M.A. Parker, High Resolution Transmission Electron Microscope Observations of Silicon Regrowth at Controlled Elevated Temperatures, Nature 322 (1986) 531533.

[34] R. Sinclair, M.A. Parker and K.B. Kim, In Situ HREM of Interface Reactions in Semiconductors, Ultramicroscopy, 23 (1987) 383-396.

[35] K. Holloway, K.B. Do and R. Sinclair, Interfacial Reactions on Annealing MolybdenumSilicon Multilayers, J. Appl. Phys., 65 (1989) 474-480.

[36] M. Chi, C. Wang, Y. Lei, G. Wang, D. Li, K.L. More, A. Lupini, L.F. Allard, N.M. Markovic, and V.R. Stamenkovic, Surface faceting and elemental diffusion behaviour at atomic scale for alloy nanoparticles during in situ annealing, Nature Comm, (2015) DOI: $10.1038 /$ ncomms 9925

[37] T. Hawa and M.R. Zachariah, Coalescence kinetics of unequal sized nanoparticles, Aeorsol Science 37 (2006) 1-15 DOI: 10.1016/j.jaerosci.2005.02.007.

[38] S. Arcidiacono, N.R. Bieri, D. Poulikakos, C.P. Grigoropoulos, On the coalescence of gold nanoparticles, Intern. J. Multiphase Flow 30 (2004) 979-994.

[39] K.Nakaso, M. Shimada, K. Okuyama, K. Deppert, Evaluation of the change in the morphology of gold nanoparticles during sintering, Aerosol Science, 33 (2002) 1061-1074.

[40] J.M. Yuk, M. Jeong, S.Y. Kim, H.K. Seo, J. Kim, J.Y. Lee, In situ atomic imaging of coalescence of Au nanoparticles on graphene: rotation and grain boundary migration, Chem. Comm. 49 (2013) 11479-11481 DOI: 10.1039/c3cc46545d.

[41] G. Hermann, H. Gleiter and G. Baro, Investigation of low energy grain boundaries in metals by a sintering technique, Acta Metall. 24 (1976) 353-9 DOI:10.1016/0001-6160(76)90010-9.

[42] E.L. Hall and J. Bentley, Energy-Filtered Imaging of Interfacial Composition in Complex Ni-Base Superalloys, J Microscopy Soc. Amer., Proc. Microscopy and Microanalysis 1995, G. 
W. Bailey, M.H. Ellisman, R. A. Hennigar, and N. J. Zaluzec, eds., (New York: Jones \& Begell, 1995) 260-1.

[43] J. Bentley, E.A. Kenik, N.D. Evans, E.L. Hall, and S.J. Zinkle, Energy-Filtered Imaging on a 300kV TEM, Electron Microscopy and Analysis 1995 (Proc. EMAG '95), Inst. Phys. Conf. Ser. No. 147, D. Cherns, ed., (Institute of Physics Publishing, Bristol, 1995) 187-190.

[44] H. Chen, Y. Yu, H.L. Xin, K.A. Newton, M.E. Holtz, D. Wang, D.A. Muller, H.D. Abruña, F.J. DiSalvo, Coalescence in the Thermal Annealing of Nanoparticles: An in Situ STEM Study of the Growth Mechanisms of Ordered Pt-Fe Nanoparticles in a $\mathrm{KCl}$ Matrix, Chem. Mater. 25 (2013) 1436-1442 DOI: 10.1021cm303489z. 